\title{
Fondements phénoménologiques de la structure prédicative
}

\author{
Par DOMINIQUE PRADELLE
}

Université de Paris 4 Sorbonne
Car les êtres [...] dont nous étions disposés à croire que les seules variations tenaient au point où nous étions placés pour les regarder, à la distance qui nous en éloignait, à l'éclairage, ces êtres-là, tandis qu'ils changent par rapport à nous, changent aussi en eux-mêmes ; et il y avait eu enrichissement, solidification et accroissement de volume dans la figure jadis simplement profilée sur la mer.
(M. Proust, La Prisonnière.)

La structure prédicative de la proposition jouit, dans la tradition logique et philosophique, d'un privilège évident depuis les analyses aristotéliciennes du De interpretatione : toute proposition énonciative est supposée reconductible, voire réductible à une proposition prédicative du type « $S$ est $p$ », attribuant à un sujet $S$ un prédicat $p^{1}$. Il a fallu les analyses de Frege, puis de Russell, pour mettre en question ce caractère paradigmatique, et lui substituer la notion de fonction propositionnelle $\mathrm{f}(\mathrm{)})$ susceptible d'être satisfaite par un argument $x$. Analysant plusieurs exemples de proposition énonciative, Frege a en effet mis en évidence l'équivoque inhérente au petit mot est dans les propositions " cette feuille est verte », " l'étoile du matin est Vénus ", " tout carré est un rectangle » et « tout triangle équilatéral est un triangle équilatéral et réciproquement ». Ce mot renvoie à la distinction fondamentale entre concept et objet, et recouvre des fonctions logiques distinctes : subsomption

\footnotetext{
${ }^{1}$ Aristote, De interpretatione, 21 b 9.
} 
d'un objet sous un concept, identification de deux objets, subordination d'un concept à un autre, équivalence de deux concepts ${ }^{1}$.

Or, malgré la réforme de la logique formelle à laquelle ont conduit de telles analyses et la construction du calcul formel des prédicats en termes fonctionnels, Husserl continue de reconnaître à la structure prédicative un statut paradigmatique ou universel. D'une part, un simple changement d'attitude subjective (Einstellungsänderung) permet de reconduire toutes les formes du discours à la forme judicative ou apophantique « $S$ est $p$ » - par exemple, le souhait direct « puisse $S$ être $p$ ! » à la proposition judicative « je souhaite que $S$ soit $p$ », etc ${ }^{2}$. D'autre part, la seconde section d'Erfahrung und Urteil, tout entière consacrée à "La pensée prédicative et les objectités d'entendement ", commence par un chapitre portant sur "Les structures générales de la prédication et la genèse des plus importantes formes catégoriales ", laissant entendre que la structure prédicative est la plus fondamentale de toutes les formes catégoriales ${ }^{3}$.

Qu'en est-il de ce primat de la structure catégoriale parmi les formes discursives et les objectités d'entendement? La phénoménologie est-elle capable de mettre en évidence le fondement de sa légitimité ? Et à quel niveau d'analyse se situent ce fondement ainsi que l'origine de la signification du est dans la structure prédicative ? Est-ce au niveau noétique des fonctions subjectives de la pensée - le est correspondant alors à une fonction synthétique, à un acte de liaison ou de séparation entre représentations subjectives, ou, plus profondément, à une orientation thématique de la subjectivité connaissante? Est-ce au niveau syntaxique du sens ou de la signification idéale — le " est » désignant alors une articulation formelle idéale entre le sens-sujet et le sens-prédicat, dépourvue de toute teneur de réalité ? Ou, enfin, au niveau ontologique de de l'objectité, c'est-à-dire de l'objet au sens large - le est ne se réduisant plus alors à une signification, mais devenant un objet idéal et formel qui se donne dans une évidence sui generis à caractériser ? Bref, si la phénoménologie doit élucider la genèse de la structure prédicative, celle-ci reconduit-elle à un fondement noétique, syntaxique ou objectal?

${ }^{1}$ G. Frege, «Über Begriff und Gegenstand », Vjschr. f. wissensch. Philosophie 16, 1892, p. 193-195.

${ }^{2}$ E. Husserl, Formale und transzendentale Logik, § 3, Hua XVII, p. 27-28 (trad. fr. S. Bachelard, Logique formelle et logique transcendantale, Paris, Puf, 1957, p. 3536).

${ }^{3}$ E. Husserl, Erfahrung und Urteil, § 47 sqq., Hamburg, Glaassen \& Goverts, 1954, p. 231 sqq. (trad. fr. D. Souche, Expérience et jugement, Paris, Puf, 1970, p. 237 sqq.). 
En outre, si le est renvoie non à un seul de ces différents niveaux, mais simultanément à tous, quelle en est la hiérarchie ? Est-il possible de poser que l'un d'eux constitue le lieu d'origine (Ursprungsort) des deux autres ? La catégorie formelle du est a-t-elle un lieu d'origine univoque ? Est-ce une origine sensible externe, renvoyant à l'inhérence de moments ou de propriétés à des objets de perception externe ? Une origine sensible interne, renvoyant à la réflexion sur l'acte de lier ensemble sujet et prédicat dans la proposition énonciative ? Ou enfin, une origine prise en un sens sui generis : non plus celui de la production, mais de la pro-duction (Hervor-Bringung) comme reconduction du moment de la forme catégoriale à son remplissement intuitif (Erfüllung) ? On est ainsi conduit à une question générale qui porte sur le sens même de la notion d'origine (Ursprung) ou de lieu originaire (Ursprungsort) des catégories en général : peut-on parler de genèse et d'origine des catégories formelles ? et en quel sens peut-on le faire, s'il ne s'agit plus de dérivation empiriste à partir de données singulières de l'expérience ?

\section{Théories traditionnelles de la prédication}

Il existe au moins une double tradition d'interprétation de l'origine des catégories dans l'histoire de la pensée. La première est la théorie de l'origine réflexive des catégories, dont les représentants notables sont Locke, ainsi qu'Arnaud et Nicole.

Pour Locke, la question philosophique par excellence est celle, génétique, de la provenance de toutes les idées, et celle-ci réside dans l'expérience ; cette dernière se laisse à son tour diviser en expérience sensible externe et en expérience réflexive ou interne - perception des objets extérieurs, et réflexion de l'esprit sur ses propres actes. Dès lors, l'énumération des types d'actes possibles sur les idées sensibles simples permet de thématiser la généalogie des idées complexes ou abstraites - combiner, joindre, séparer. Appliquons ce schéma généalogique réflexif à l’idée exprimée par la copule est : celle-ci a une origine noétique, à savoir dans la réflexion portant sur l'acte mental de formation des propositions ou des jugements - acte qui consiste à joindre ou séparer des idées (propositions mentales) ou des mots (propositions verbales) ${ }^{1}$. On est ainsi renvoyé à une double couche d'actes : tout d'abord, l'acte de joindre sujet et prédicat dans

1 J. Locke, Essay on Human Understanding, IV, chap. V, § 5, éd. P. H. Nidditch, Oxford, Clarendon Press, 1975, p. 575-576 (trad. fr. J.-M. Vienne, Essai sur l'entendement humain, Livres III et IV, Paris, Vrin, 2006², p. 348).

56

Bull. anal. phén. XII 2 (2016)

http://popups.ulg.ac.be/1782-2041/ @ 2016 ULg BAP 
la proposition affirmative et de les séparer dans la proposition négative, cette jonction (synthesis) et cette séparation (diairesis) étant des opérations situées dans l'esprit et opérées par lui ; puis la réflexion sur ces opérations, qui est une perception interne de ses propres actes par l'esprit. Est et n'est pas désignent donc non des formes d'appartenance, d'inhérence ou de convenance de propriétés ou de moments à des objets — c'est-à-dire des structures ontologiques résidant dans l'étant lui-même -, mais des opérations psychiques ou des actes noétiques.

De même, la Logique de Port-Royal d'Arnaud et Nicole reconduit la structure binaire et prédicative de toute proposition énonciative à la nature synthétique ou diairétique de la formation subjective du jugement simple ${ }^{1}$. C'est là une tradition logique fondée sur l'assomption d'un passage célèbre du livre E de la Métaphysique d'Aristote qui, à la copule est, attribue le sens exclusivement noétique d'une opération de la pensée liant ou séparant les termes :

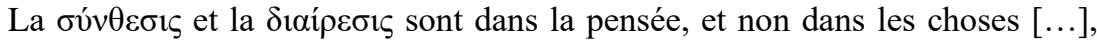
car la pensée réunit ou sépare, pour un sujet donné, soit une essence, soit une certaine qualité, soit une certaine quantité, soit tout autre mode ${ }^{2}$.

Cependant, il existe une autre lignée interprétative de la copule est, fondée cette fois sur une compréhension ontologique - et non plus noétique - de la $\sigma u ́ v \theta \varepsilon \sigma ı \iota_{\varsigma}$ et de la $\delta 1 \alpha i ́ \rho \varepsilon \sigma 1 \varsigma$, et rattachée à un passage non moins célèbre du livre $\Theta$ de la Métaphysique :

L'à $\lambda \eta \theta \dot{\varepsilon} \zeta$ et le $\psi \varepsilon \tilde{\delta} \delta$ o $\varsigma$ consistent, au regard des étants, dans le tenir-d'emblée-

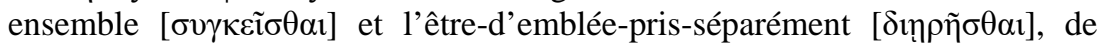
sorte que dire le vrai, c'est prendre ce qui est séparé dans son être-séparé, et ce qui tient ensemble dans son tenir-ensemble ${ }^{3}$.

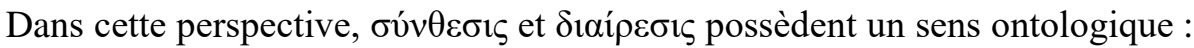
celui de l'unité, de l'être-ensemble ou de la cohésion entre sujet et prédicat,

\footnotetext{
${ }^{1}$ A. Arnaud \& P. Nicole, Logique de Port-Royal, II, chap. III : « [la proposition] doit avoir deux termes, l'un, de qui l'on affirme ou de qui l'on nie, lequel on appelle sujet; et l'autre, que l'on affirme ou que l'on nie, lequel s'appelle attribut ou praedicatum.

Et il ne suffit pas de concevoir ces deux termes ; mais il faut que l'esprit les lie ou les sépare. Et cette action de notre esprit est marquée dans le discours par le verbe est. » ${ }^{2}$ Aristote, Métaphysique, E, 4, 1027 b 30.

${ }^{3}$ Aristote, Métaphysique, $\Theta, 10,1051$ b 2-5.
} 
ou au contraire de leur exclusion ou incompatibilité - à savoir une connexion ontologique, située à même l'étant et déclinée selon les différentes manières que présente un attribut catégorial (selon la quantité, la qualité, le lieu, le temps, etc.) d'être dans le sujet (essentiellement ou accidentellement,

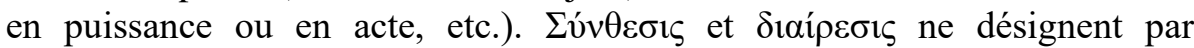
conséquent plus des modalités noétiques, des actes de la pensée, mais c'est au contraire l'étant lui-même qui est pensé à partir du syn- de la synthesis, c'est-à-dire de la cohésion entre ses moments, parties, composantes eidétiques ou accidentelles ; la signification du est réside par conséquent au niveau ontologique, dans l'inesse du praedicatum inest subjecto envisagée selon ses diverses modalités. Locke lui-même, dans sa définition de la vérité comme liaison ou séparation des signes correspondant à la convenance ou disconvenance des choses entre elles, fait bel et bien référence à la convenance ou à l'accord objectal, au sein des choses mêmes, entre sujet et prédicat ${ }^{1}$. Et, au $\S 19$ de la déduction transcendantale des catégories, Kant note le caractère insatisfaisant de la définition du jugement (Urteil) comme représentation d'un rapport entre deux concepts, du fait du caractère indéterminé d'un tel rapport ; il est au contraire nécessaire de le déterminer en référence à l'unité objective de l'aperception transcendantale, c'est-à-dire de ressaisir la fonction de la copule est comme étant de «distinguer l'unité objective des représentations données de leur unité subjective » - objective, parce qu'il s'agit d'une synthèse des intuitions régie par les principes de validité du rapport dans l'objet lui-même ${ }^{2}$ : ce qui est en jeu, c'est certes l'acte de synthèse ou de liaison des représentations dans le jugement, mais c'est aussi et surtout le principe de leur validité dans l'objet lui-même, et non dans les seuls états internes du sujet.

\section{Refus husserlien de l'interprétation noétique de la copule est au profit de l'interprétation ontologique}

Quelle est, au regard de cette double lignée interprétative, la position phénoménologique?

1 J. Locke, Essay on Human Understanding, IV, chap. 5, § 2: «L'union ou la séparation des signes selon que les choses signifiées par eux conviennent ou non les unes avec les autres » (éd. Nidditch, 574 ; trad. fr., p. 345).

${ }^{2}$ I. Kant, Kritik der reinen Vernunft [KrV], Transz. Analytik, Deduktion der reinen Verstandesbegriffe, § 19, B 140 sqq. (trad. fr. Critique de la raison pure, DelamarreMarty, Paris, Gallimard folio, 1980, p. 520, A. Renaut, Paris, GF-Flammarion, 2006, p. 533). 
Dans la Sixième Recherche, Husserl s'oppose radicalement à toute genèse purement noétique et réflexive de la copule est, qui reconduirait cette dernière à un acte psychique donné dans la perception interne :

L'origine du concept d'être et des autres catégories [Ursprung des Begriffes Sein und der übrigen Kategorien] ne réside pas dans le domaine de la perception interne [nicht im Gebiete der inneren Wahrnehmung] ${ }^{1}$.

Ce n'est pas dans la réflexion sur des jugements ou plutôt sur des remplissements de jugements, mais dans les remplissements de jugements eux-mêmes [in den Urteilserfüllungen selbst] que réside véritablement l'origine des concepts d'état de choses et d'être [Ursprung der Begriffe Sachverhalt und Sein] (au sens de la copule); ce n'est pas dans ces actes en tant qu'objets, mais dans les objets de ces actes que nous trouvons le fondement de l'abstraction pour la réalisation desdits concepts ${ }^{2}$.

On conçoit aisément la portée négative et critique de telles thèses : l’origine du concept d'être ou de la copule est ne réside pas dans les actes de jugement subjectifs, ni dans la réflexion ou perception interne par laquelle sont présents à l'esprit du sujet ses propres actes judicatifs ; bref, la signification du est ne résulte pas de la réification d'une fonction noétique interne. Mais quel est alors le sens positif de ces thèses ? Que signifie le fait que le concept d'être trouve son origine dans le remplissement du jugement lui-même, et non plus dans la réflexion sur les jugements et leur remplissement ? Pour répondre à une telle question, il convient de suivre dans le détail l'argumentation de Husserl.

Posant le «problème du remplissement des formes de signification catégoriales » (Erfüllung kategorialer Bedeutungsformen ${ }^{3}$ ), Husserl pose une double question relative au rapport entre intention et intuition : d'une part, celle de la possibilité de remplissement intuitif des énoncés complets (Erfüllung der ganzen Aussagen ${ }^{4}$ ), qui conduit à l'idée de leur remplissement grâce à la donation évidente de l'état de choses (Sachverhalt) catégorial que $S$ est $p$, lui-même fondé sur la perception de la chose $S$ et la saisie de l'état de fait ; d'autre part, celle de la possibilité de remplissement intuitif des parties ou

${ }^{1}$ E. Husserl, Logische Untersuchungen [LU], VI. Untersuchung, § 44, Hua XIX/2, p. 667 (trad. fr. Élie-Kelkel-Schérer, Recherches logiques, tome III, Paris, Puf, 1974, p. 173).

${ }^{2}$ E. Husserl, LU, VI. Unt., § 44, Hua XIX/2, 669-670 (trad. fr., p. 174).

${ }^{3}$ E. Husserl, LU, VI. Unt., § 40, Hua XIX/2, 657 (trad. fr., p. 159).

${ }^{4}$ E. Husserl, LU, VI. Unt., § 40, Hua XIX/2, 658 (trad. fr., p. 160). 
moments du logos auxquels ne correspond aucune matière, c'est-à-dire aucun contenu de signification ou de connaissance possible qui renvoie à une teneur au sein des objets mêmes — c'est la question du remplissement possible des moments de pure forme catégoriale de signification, du type et, ou, un, tous les, est ${ }^{1}$.

Quel est le fil conducteur permettant de répondre à cette question ?

Il réside dans le caractère paradigmatique que possède le rapport entre la visée d'une signification propre (Eigenbedeutung) et les perceptions qui viennent la remplir ${ }^{2}$ - caractère qui en autorise l'universalisation, c'est-àdire l'extension à tous les moments de la signification en général : si la visée de la signification Cologne se remplit dans la perception de la ville ellemême, il doit y avoir un remplissement — donc une donation - qui corresponde à tous les moments de la signification, y compris purement formels.

Suit la distinction de statut entre forme et matière propositionnelles et, corrélativement, entre le remplissement de la forme et celui de la matière. Dans des propositions du type « $S$ est $p$ », « quelque $S$ est $p$ », « cet $S$ est $p$ », « tous les $S$ sont $p$ », etc., c'est exclusivement aux significations revenant aux symboles littéraux $S$ et $p$ que correspond quelque chose dans l'objet luimême; et ce sont donc exclusivement de telles significations qui sont susceptibles de se remplir directement (direkt) dans la perception de l'objet - tandis que les moments de la forme catégoriale (quelque, cet, tous les) ne correspondent à rien dans les objets mêmes, et n'admettent par principe aucun remplissement direct dans la perception ${ }^{3}$. Il existe pour la matière de la signification une possibilité de remplissement direct, parce qu'il lui correspond un moment réal dans l'étant, mais en revanche nulle possibilité de ce type pour la forme catégoriale, parce qu'il n'y a dans son cas aucune concordance entre la forme signifiée et quelque moment réal à même les objets. Or, il en est ainsi pour l'être, qui n’est pas un prédicat de réalité :

Je puis voir la couleur [Farbe], mais non ce qu'est l'être-coloré [nicht Farbigsein]. Je puis sentir le poli [Glätte], mais non ce qu'est l'être-poli [nicht aber

${ }^{1}$ E. Husserl, $L U$, VI. Unt., § 40, Hua XIX/2, 658 (trad. fr., p. 160-161).

${ }^{2}$ E. Husserl, $L U$, VI. Unt., $\S 40$, Hua XIX/2, 659 : « Le prototype pour l'interprétation du rapport entre les actes de signification et d'intuition serait donc fourni par le rapport de la signification propre avec les perceptions correspondantes. » (trad. fr., p. 161).

${ }^{3}$ E. Husserl, LU, VI. Unt., § 42, Hua XIX/2, 663-664 (trad. fr., p. 167).

60 
das Glatt-sein]. Je puis entendre le son [den Ton], mais non ce qu'est l'êtresonore [nicht aber das Tönend-sein $]^{1}$.

D’où la double conséquence que :

L'être n'est rien dans l'objet [nichts im Gegenstande], n'en est pas une partie [kein Teil desselben], ni un moment qui lui soit inhérent [kein ihm einwohnendes Moment], [...], ni un caractère constitutif [kein konstitutives Merkmal], de quelque manière qu'on le conçoive.

et que

l'être n'est rien non plus à même l'objet [auch nichts an einem Gegenstande], [...] et n'est donc pas un caractère au sens « réal » [im realen Sinne überhaupt kein "Merkmal”]2.

Ainsi, l'être n'est ni un fragment réal de l'objet (une partie au sens strict, comme la tête du cheval l'est du cheval), ni un moment abstrait de l'objet (comme la couleur relativement à une étendue colorée), ni une relation ou une forme d'unité réale qui soit inhérente aux contenus perceptifs euxmêmes (par exemple, de l'ordre des relations temporelles, spatiales, causales ou de la gradation des intensités). Dans la typologie générale des concepts présentée au $\S 60$ de la Sixième Recherche, il en découle que l'être n'est ni un concept sensible (sinnlicher Begriff : rouge, coloré, etc.), ni un concept mêlé de formes catégoriales (mit kategorialen Formen vermischter : droite, cercle, etc.), mais un concept purement catégorial (rein kategorialer Begriff), absolument non réal ou non contentuel ${ }^{3}$.

Une fois reconnu ce statut purement formel de la copule est, il devient possible de mener l'analyse du remplissement de sa visée : si le Bedeuten désigne l'acte de signifier et de comprendre le sens du est, quand et comment

${ }^{1}$ E. Husserl, LU, VI. Unt., § 43, Hua XIX/2, 666 (trad. fr., p. 169). C’est là une reconduction de la thèse kantienne selon laquelle "Sein ist offenbar kein reales Prädikat », l'être n'est manifestement pas un prédicat réal, exprimant la teneur de réalité des choses ( $K r V$, Transz. Dialektik, A 598/B 626, trad. fr. DM, p. 520, AR, p. 533). Il est fâcheux que les traductions françaises disponibles traduisent toutes "real » par réel, ce qui suscite une confusion avec wirklich, effectif, qui est une catégorie de la modalité : l'être relève de la Wirklichkeit et, à ce titre, n’appartient pas à la realitas de l'objet, c'est-à-dire à son essence ou à sa possibilité — laquelle n’est justement pas sa réalité, entendue comme existence !

${ }^{2}$ E. Husserl, LU, VI. Unt., § 43, Hua XIX/2, 666 (trad. fr., p. 169-170).

${ }^{3}$ E. Husserl, LU, VI. Unt., § 60, Hua XIX/2, 713 (trad. fr., p. 221).

61 
passe-t-on de cette compréhension de la signification verbale à la donation de l'objet formel correspondant? Pourquoi est-on contraint de transcender le niveau du sens et de sa compréhension, pour passer à celui de l'objectualité et de sa donation intuitive ? Y a-t-il quelque objet idéal et formel qui puisse s'attester dans une évidence spécifique, et qui soit irréductible à la fonction syntaxique que possède la copule dans toute forme de discours prédicatif ?

Un principe fondamental de la phénoménologie husserlienne relativement à la signification est que toute signification trouve son origine, c'est-àdire fondement et légitimité, dans une évidence où elle n'est pas seulement objet de compréhension, mais assume une fonction de connaissance ou de dévoilement de l'objet ${ }^{1}$.

Un second principe oppose la forme de remplissement respective des significations catégorématiques et syncatégorématiques, indépendantes et dépendantes, closes sur soi et requérant un complément de signification, et soumet ce dernier à une exigence de contextualité. Les significations non autonomes sont en effet des contenus fondés qui ne peuvent exister isolément, mais doivent prendre place à titre de contenus partiels dans une totalité signifiante indépendante ${ }^{2}$; en conséquence, le remplissement d'une signification formelle insaturée comme et, ou, est ou tous les ne peut avoir lieu qu'au sein du remplissement d'une proposition prédicative où elle intervient, c'est-à-dire de la connaissance d'un état de choses syntaxiquement mis en forme par cette catégorie formelle :

Des syncatégorèmes détachés, comme égal, en liaison avec, et, ou, ne peuvent devenir objets de compréhension intuitive, acquérir un remplissement de signification, si ce n'est dans le contexte d'une intention de signification plus vaste [im Zusammenhang eines umfassenderen Bedeutungs-zusammenhang] $]^{3}$.

Nulle signification syncatégorématique, c'est-à-dire nul acte d'intention de signification dépendante, ne peut assumer de fonction de connaissance, sinon dans le contexte d'une signification syncatégorématique ${ }^{4}$.

${ }^{1}$ E. Husserl, $L U$, Einleitung, § 2, Hua XIX/1, 10 (trad. fr. Élie-Kelkel-Schérer, Recherches logiques, tome II/1, Paris, Puf, $1969^{2}$, p. 6).

${ }^{2}$ E. Husserl, LU, IV. Unt., § 7, Hua XIX/1, 319-320 (trad. fr. Élie-Kelkel-Schérer, Recherches logiques, tome II/2, Paris, Puf, $1972^{2}$, p. 104-106).

${ }^{3}$ E. Husserl, $L U$, IV. Unt., § 9, Hua XIX/1, 323 (trad. fr., p. 108).

${ }^{4}$ Ibid. 
Ou bien le syncatégorème détaché n’a pas du tout la même signification que dans un contexte catégorématique, ou bien il a la même signification, mais reçoit un complément de signification, ce dernier fût-il tout à fait indéterminé dans son contenu [eine wenn auch sachlich ganz unbestimmte Bedeutungsergänzung $]^{1}$.

Prenons un exemple. La signification de la conjonction et peut certes être comprise isolément ; cependant, elle n'est pleinement comprise et n'assume de fonction de connaissance que lorsqu'elle est employée dans une proposition complète qui s'avère adéquate à un état de choses qu'elle fait connaître - c'est-à-dire lorsqu'elle est appliquée à une collection concrète d'objets singuliers qui sont subsumés sous un même concept : par exemple, dans la proposition « Mercure, Vénus, la Terre et Mars sont des satellites du soleil ». Le remplissement de la visée de tels syncatégorèmes formels possède donc une structure holistique soumise au principe de contextualité intuitive : c'est dans le cadre de l'évidence globale d'un état de choses que se remplit la visée des catégories formelles. Quelle est donc la nature du complément de signification de contenu indéterminé qui advient alors ? On le comprend sur l'exemple mentionné : lorsque le et s'applique aux quatre planètes mentionnées, se constitue alors l'état de choses « que ces planètes sont des satellites du soleil » et, par voie de conséquence, l'ensemble de ces planètes comme sous-ensemble des satellites du soleil, ou de l'extension du concept « satellite du soleil »; par là, la fonction syntaxique du et, implicite lorsque la conjonction était comprise isolément, est actualisée sur son versant apophantique par l'évidence de l'état de choses et, sur son versant ontologique, par la donation de l'ensemble des planètes. La forme syntaxique et possède ainsi une double fonction: mise en forme conjonctive de l'état de choses, et structuration ontologique fondée de l'objectité ensemble ${ }^{2}$; et si le complément de signification demeure indéterminé quant à sa matière, c'est simplement parce qu'il est dépourvu de contenu réal, et qu'il consiste uniquement dans l'actualisation de la fonction syntaxique susdite au double niveau apophantique et ontologique.

${ }^{1}$ E. Husserl, LU, IV. Unt., § 9, Hua XIX/1, 324 (trad. fr., p. 109).

2 E. Husserl, Form. u. transz. Logik, § 42d, Hua XXVII, 119-120 : « Assurément, il est ici tout d'abord déroutant que les opérations syntaxiques aient une double fonction: d'un côté comme créatrices de formes, par le fait qu'elles créent les objectités qui ont diverses formes syntaxiques, des formes dérivées du quelque chose en général [...]. De l'autre, elles ont pour fonction d'être les différentes syntaxes que peut prendre une telle objectité catégoriale » (trad. fr., p. 155). 
Toute cette analyse se laisse appliquer à l'exemple de la copule est : dans la compréhension purement verbale de la proposition "l'or est jaune », le est est un moment de signification (Bedeutungsmoment) insaturé, qui est uniquement signifié (nur bedeutet) ou visé de manière purement signitive (signitiv gemeint) ; mais

il est lui-même donné [selbst gegeben] ou, du moins, présumé donné [vermeintlich gegeben] dans le remplissement qui, éventuellement, s'ajuste au jugement : dans l'aperception de l'état de choses présumé [Gewahrwerdung des vermeinten Sachverhalts] $]^{1}$.

En d'autres termes, lorsque le est est employé dans le contexte de la connaissance de l'état de choses "que l'or est jaune ", il y a une évidence donatrice de cet état de choses, donc un remplissement de la visée signitive ou compréhensive de la proposition «l'or est jaune»; ce n'est plus seulement la proposition qui est comprise, mais le fait ou l'état de choses qui est connu et attesté ; or, ce remplissement global du Sachverhalt implique celui de toutes les composantes réales et syntaxiques de la proposition, donc la donation des objectités correspondant aux divers moments de la signification, que ces derniers soient réals ou syntaxiques. Par conséquent, il doit y avoir une donation de l'objectité catégoriale correspondant à la seule forme syntaxique est, c'est-à-dire une évidence du est lui-même comme objet de degré supérieur : ce dernier n’est pas seulement visé et compris dans sa signification verbale, mais donné à titre d'objet. Quant au supplément de signification qui advient lors de ce remplissement contextualisé, il reste indéterminé en son contenu - puisque la copule ne correspond à aucun caractère dans l'objet : ni propriété, ni moment, ni relation, ni forme d'unité - , et ne consiste que dans l'actualisation de la fonction syntaxique du est au sein de l'état de choses.

Comme dans le cas de la conjonction, ce supplément possède deux versants. D'une part, la forme catégoriale de la copule est ici entendue au niveau ontologique: alors que pour la simple compréhension du sens la copule désignait le mode de liaison apophantique entre sujet et prédicat, dans la connaissance elle intervient comme un constituant du Sachverhalt, et désigne le mode ontologique de cohésion qui relie le substrat à ses propriétés ou relations. D'autre part, le plan ontologique se dédouble en un niveau fondateur (celui de la réceptivité) et un niveau fondé (celui de l'entendement) : le Sachverhalt (état de choses) n'étant que l'explicitation thématique

${ }^{1}$ E. Husserl, $L U$, VI. Unt., § 44, Hua XIX/2, p. 668 (trad. fr., p. 172). 
d'une Sachlage (situation) elle-même fondée dans le mode d'être de l'objetsubstrat ${ }^{1}$, le est renvoie au mode de cohésion singulier entre les parties et le tout au sein du substrat en question - ici, l’inhérence spécifique du moment abstrait « jaune » dans le substrat perceptif « or ».

Tirons-en les conclusions essentielles.

1/ Plusieurs distinctions ont été ainsi opérées. D’abord, entre les versants noétique et noématique : le est ne désigne pas l'acte noétique de synthèse entre les représentations de sujet et de prédicat, ou l'opération noétique de jonction entre des concepts, mais l'être-lié (Verbundenheit) noématique entre sujet et prédicat, substrat et propriété. Ensuite, une distinction entre les niveaux de la signification (bedeutungsmäßig) et de l'objet (gegenständlich): le est désigne un syn, une Verbundenheit non seulement entre les significations-sujet et -prédicat, mais entre l'objetsubstrat (référence ou dénotation : l'objet à propos duquel on juge) et l'objetou moment-propriété. Enfin, une distinction entre aspect matérial et formel : le est ne se confond avec aucune connexion matériale singulière ou spécifique entre substrat et propriété (inhérence d'une couleur à une étendue, d'une forme à un objet, d'un nombre à une pluralité...), mais désigne un être-lié ontologique-formel, c'est-à-dire le type de Verbundenheit qui englobe tous ces rapports possibles d'inhérence, d'appartenance, de comparaison relationnelle, de subsomption, etc., et en même temps trouve en eux ses fondements.

2/ La seconde conclusion concerne la notion d'origine (Ursprung) ou de genèse (Entspringen) des concepts d'être et d'état de choses : l'origine, comme surgissement ou naissance de la catégorie d'être, n'est nullement une production de son concept par quelque opération intellectuelle. "Entspringen », écrit Husserl entre guillemets, c'est « nous être donné soi-même sur le fondement d'un acte $»^{2}$. La catégorie Sein se situe sur le versant noématique, objectal ; l'acte donateur qu'est l'intuition catégoriale n'est que le corrélat noétique de l'être, son mode de donation ou de reconduction à l'évidence au sein d'un état de choses. L'acte de liaison prédicative n'est donc pas une production de la forme catégoriale (ici, prédicative), mais seulement la condition de possibilité subjective de son venir-à-l'apparaître ; il n'est par conséquent que la condition subjective de la pro-duction (Hervor-

${ }^{1}$ E. Husserl, Erf. u. Urt., § 59, p. 285-286 (trad. fr., p. 288-289).

2 E. Husserl, LU, VI. Unt., § 44, Hua XIX/2, 670 : «De même qu'un concept quelconque ne peut "naître", c'est-à-dire nous être donné lui-même, que sur le fondement d'un acte [uns selbst gegeben werden auf Grund eines Aktes] » (trad. fr., p. 174). 
Bringung) de la catégorie, c'est-à-dire de sa reconduction à l'évidence ; c'est l’Idée générale d'acte qui correspond à l'Idée formelle de la catégorie.

\section{Analyse husserlienne de la forme prédicative au niveau de la signification}

Ce privilège du jugement prédicatif dans la tradition logique est-il légitime ? S'il est attesté par l'élucidation de l'évidence des états de choses, l'est-il également, au niveau du sens, par l'analyse des moments de signification de la proposition ? D'une part, le jugement prédicatif est-il la formation logique originaire, c'est-à-dire la proposition la plus simple et la forme universelle dont sont dérivées, par transformation et composition, toutes les formes propositionnelles ? D'autre part, quel est le sens de la copule est dans cette articulation propositionnelle des significations ?

Il est ici nécessaire de confronter la thèse husserlienne aux critiques fondamentales qu'adressent Brentano et Frege au primat de la proposition prédicative.

Brentano nie que l'essence du jugement en général présente un caractère synthétique et prédicatif ; et il justifie cette thèse négative par une méthode générale de réduction ou de traduction des jugements: il est possible de réduire ou traduire tout jugement catégorique de type " $S$ est $p$ » (et ses variantes négatives et quantifiées) en une proposition existentielle de type « $\exists S p$ »; toute proposition prédicative s'avère ainsi traduisible en une position affirmant ou niant la validité d'une représentation complexe comprenant un sujet et un (ou plusieurs) prédicat ${ }^{1}$.

Quant à Frege, il critique les notions de sujet et de prédicat en logique, ainsi que la conception de l'unité propositionnelle comme liaison copulative (grâce au est) entre un sujet et un prédicat ; cette décomposition recouvre en effet la différence de fonction logique du " est », ainsi que la nature effective des parties du sens propositionnel et de leur composition — à savoir l'égalité, qui est réversible («l'étoile du matin est Vénus »), ou la subsomption d’un objet sous un concept, qui est irréversible («l'étoile du matin est une planète »), ou la subordination d'un concept à un autre, également irréversible («tout carré est un rectangle»). Le principe fondamental de la composition des unités de sens réside dans la distinction entre sens insaturé

${ }^{1}$ F. Brentano, Psychologie vom empirischen Standpunkte, Zweites Buch, Kap. VII, $\S 5$, 1874, 48-49 (trad. fr. M. de Gandillac rev. J.-F. Courtine, Psychologie du point de vue empirique, Paris, Vrin, 2008, p. 228).

66 
et saturé qui, au niveau de la dénotation, renvoie à la distinction entre concept et objet, fonction $\mathrm{f}()$ ou $\mathrm{p}(S)$ - et non plus « $S$ est $p$ » - et argument (variable) $x$. Ainsi, d'une part, la nature prédicative du concept n'est pas niée, mais assimilée au caractère insaturé (ungesättigt) de la fonction, au fait qu'elle requiert un complément (ergänzungsbedürftig) pour désigner une valeur de vérité, faisant par conséquent disparaître la copule est comme opérateur de liaison entre des termes ; d'autre part, cette distinction entre saturation et insaturation opère au double niveau du sens (sens clos vs fonctionnel) et de la dénotation (dénotation objectale vs. conceptuelle) ${ }^{1}$.

Quelle est à ce sujet l'analyse husserlienne ? Quelle thèse le recours intuitionniste à l'évidence donatrice permet-il d'attester ?

Suivons les différents points de l'argumentation de Husserl.

1/ Aux significations se laisse tout d'abord appliquer la distinction ontologique-formelle entre Selbständigkeit (indépendance, autosubsistance) et Unselbständigkeit (dépendance, non-autosubsistance).

Étant une sorte particulière d'objets - des objets idéaux -, les significations se divisent elles aussi en concreta et abstracta, contenus indépendants et dépendants. Les significations du premier type se caractérisent par l'être-pour-soi (Für-sich-Sein) ou le pouvoir-être-données-pour-soi (Fürsich-gegeben-können), c'est-à-dire les deux critères cartésiens de la substantialité, indépendance ontologique (être en soi) et épistémique (être concevable isolément) ${ }^{2}$, reformulés en termes phénoménologiques (être donnable isolément); celles du second type, par le Nur in einem umfassenden Bedeutungszusammenhang sein können (ne pouvoir être données que dans un contexte plus large de signification) ${ }^{3}$; à la compréhensibilité indépendante ou isolée des premières s'oppose la compréhensibilité exclusive au sein d'un contexte de signification. Or, en toute rigueur, la Selbständigkeit ne vaut que pour les seules propositions énonciatives entières ${ }^{4}$. Il en découle un principe

${ }^{1}$ G. Frege, «Über Begriff und Gegenstand », Vjschr. f. wissensch. Philosophie, 16, 1892, 193 sqq. (trad. fr. Cl. Imbert, «Concept et objet», in Écrits logiques et philosophiques, Paris, Seuil, 1971, p. 128 sqq.).

${ }^{2}$ R. Descartes, Les principes de la philosophie, I, art. 51, 52 et 60, AT IX, II, 47 et 51.

${ }^{3}$ E. Husserl, Alte und neue Logik. Vorlesung 1908/09, Einleitung, Hua Materialien VI, 59. Logik und allgemeine Wissenschaftstheorie 1917/18, § 23, Hua XXX, 102-104.

${ }^{4}$ E. Husserl, Alte und neue Logik, Hua Mat. VI, 60. Logik 1917/18, § 23, Hua XXX, 104 : «Toute signification indépendante est une proposition [jede selbständige Bedeutung ist ein Satz], et, dans la sphère apophantique qui est pour nous en 
de contextualité propositionnelle pour tout élément de signification : c'est uniquement à partir de la proposition assertorique entière et par la fonction qu'il y occupe que l'on peut comprendre un constituant sémantique ou syntaxique - ce qui vaut eo ipso pour la copule est. Comme le dit Frege, il faut partir de la proposition et procéder par analyse, et non des constituants ultimes ou atomes de sens pour constituer à partir d'eux la proposition par synthèse ${ }^{1}$.

2/ La méthode analytique permet de distinguer deux sens de la syntaxe et des notions de matériau et forme syntaxiques.

On peut, en effet, considérer les constituants (Glieder) d'une proposition dans une double perspective. La première permet de dégager la distinction entre matériau et forme de signification (Bedeutungsstoff vs. -form) : un matériau syntaxique (comme Socrate, roi, lion, vert, semblable) comporte une référence aux choses (Sachbezüglichkeit) ou une teneur de chose (Sachhaltigkeit), c'est-à-dire la relation à des Sachen relevant d'un domaine déterminé ; ces matériaux sont toujours donnés dans une forme syntaxique déterminée, à savoir la fonction de pensée (Denkfunktion) qui lui revient au sein de la proposition entière - forme de sujet, d'objet, de prédicat, d'attribut, de proposition antécédente ou conséquente, etc. - , le même matériau pouvant l'être ne varietur dans diverses fonctions syntaxiques ${ }^{2}$. Mais le procès d'analyse aboutit aussi à la distinction entre matériau pur et informé (reiner und geformter Stoff), matériau et forme nucléaires (Kernstoff vs. -form) ; et, en dernière instance, l'analyse conduit à des constituants ultimes (letzte Glieder), simples ou non divisibles (einfach, nicht mehr zu zergliedern), qui se répartissent en différentes catégories fondamentales de syntagmes (substantivité, adjectivité, relationalité : les trois formes nucléaires) ; quant au matériau nucléaire, il désigne la pure teneur de chose (Sachgehalt) en laquelle congruent différentes mises en forme nucléaire (p. ex. l'élément commun à l'adjectif ähnlich, au substantif Ähnlichkeit et à la relation Ähnlichsein, ou bien à rot et Röte) ${ }^{3}$. Le premier concept de syntaxe désigne ainsi la fonction syntaxique au sein d'une proposition; le

question, une proposition assertorique [ein behauptender Satz], un jugement entendu au sens noématique. »

${ }^{1}$ G Frege, [Aufzeichnungen für Ludwig Darmstaedter], in Nachgelasssene Schriften, Hamburg, F. Meiner, 1969, 273 : « Je ne pars pas des concepts pour composer avec eux la pensée ou le jugement, mais j'obtiens les parties d'une pensée à partir d'une décomposition de celle-ci. » (trad. fr. Ph. de Rouilhan et Cl. Tiercelin, Écrits posthumes, Nîmes, J. Chambon, 1994, p. 299).

${ }^{2}$ E. Husserl, Logik 1917/18, § 24a, Hua XXX, 106-108.

${ }^{3}$ E. Husserl, Logik 1917/18, § 25, Hua XXX, 111-112. 
second, les éléments formels ultimes qui appartiennent à une teneur de signification matériale.

3/ De là résulte la possibilité d'élucider la décomposition de la proposition en fonction de ce double niveau syntaxique, et de déterminer avec précision le concept logique traditionnel de terme. Barbara :

Prenons, en effet, une forme syllogistique traditionnelle comme

« tous les $A$ sont $B$

or tous les $B$ sont $C$

donc tous les $A$ sont $C$ ».

Le terme $B$, qui apparaît une fois sous la forme-sujet, et l'autre sous la forme-prédicat, désigne le matériau syntaxique commun à ces fonctions syntaxiques ; mais si, au niveau plus profond de la syntaxe, il apparaît une fois sous la forme substantive, et l'autre sous la forme adjective, $B$ désigne alors le matériau nucléaire commun à ces formes nucléaires. Par conséquent, le concept logique traditionnel de terme (terminus) doit être reconduit à celui de matériau nucléaire abstrait, totalement dépourvu de forme syntaxique ${ }^{1}$; et, tout matériau nucléaire étant une unité de signification relativement indépendante, la loi de composition qui en résulte est la suivante :

Le jugement propositionnel simple [propositional-einfaches Urteil] doit être formé au moins à partir de deux significations simples indépendantes [aus mindenstens zwei einfach-selbständigen Bedeutungen gebildet sein] pour être une signification de pensée complète ${ }^{2}$.

Sur fond d'une telle décomposition en matériaux ultimes, on conçoit alors quelle est la fonction de la copule est: elle désigne la forme de pensée (Denkform) ou de connexion (Verknüpfungsform) qui est nécessaire pour relier des matériaux nucléaires clos sur eux-mêmes, et spécifique à un type de proposition (Satztypus) dont les termes appartiennent à des catégories syntaxiques déterminées - par exemple, la forme de connexion entre un sujet substantif et un prédicat adjectif, ou encore, entre un sujet substantif, un prédicat relationnel et un relatif substantif. De là résulte la justification du privilège traditionnel de la forme prédicative ; l'analyse de la proposition doit en effet se faire en termes copulatifs, et non fonctionnels comme l'affirme Frege : à savoir « $S$ / est / p », et non « être- $p(S)$ » pour l'expression d'une

${ }^{1}$ E. Husserl, Logik 1917/18, § 25, Hua XXX, 114.

2 E. Husserl, Logik 1917/18, § 28c, Hua XXX, 130. 
prédication simple ; et " $\mathrm{S} /$ est / $R$ vis-à-vis de $S$ ' », et non « être- $R(\mathrm{~S}, S$ ') » pour l'expression d'une prédication relationnelle (le trait oblique / marquant la césure au sein du sens propositionnel). C'est donc la poursuite de l'analyse syntaxique jusqu'aux constituants nucléaires dépourvus de syntaxe qui implique la nécessité de la copule prédicative est comme forme de liaison entre éléments saturés.

4/ Une objection triviale à ce primat de la forme copulative vient pourtant à l'esprit.

S'il existe une distinction entre les fonctions syntaxiques ultimes (formes nucléaires substantive, adjective et relationnelle), n'est-ce pas justement cette différence de forme entre les syntagmes élémentaires qui fonde la distinction entre les diverses formes propositionnelles (p. ex. les propositions prédicative et relationnelle) - ce qui rendrait inessentielle, voire redondante la copule " est " ? Le fait que les matériaux nucléaires ultimes se présentent toujours comme pourvus de forme nucléaire - et jamais à l'état de totale nudité logique - , et que ces formes nucléaires — ou, comme dit Husserl dans la Quatrième Recherche, les catégories de signification (Bedeutungskategorien $)^{1}$ — prescrivent par elles-mêmes les lois de leur enchaînement, ne rend-il pas tout à fait superflu le « est » ?

Conscient de cette possibilité, Husserl fait la distinction entre deux manières de concevoir le prédicat.

D’un côté, on a le prédicat complet (Vollprädikat) « est p », de l'autre, le concept de prédicat (Prädikatbegriff) ou le prédicat au sens restreint (engeres Prädikat) $p$; le premier désigne le tout de ce qui est énoncé du sujet (das Ganze von $S$ Ausgesagte), le second, le terminus au sens traditionnel ${ }^{2}$. Où se trouve à présent la césure véritable au sein de la proposition prédicative ? Elle se situe entre le sujet et le prédicat complet, selon le schéma « $S$ / est $p »^{3}$, ce qui implique une division en deux niveaux (Zweistufigkeit) : à savoir le soubassement que constitue le sujet (vं

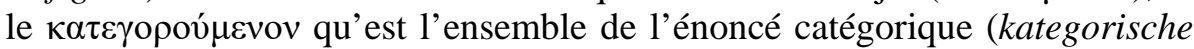
Aussage). Cela aurait justement dû conduire Husserl à adopter l'analyse de Frege, et à décomposer la structure propositionnelle en un constituant saturé (le Subjekt-Glied S) et un constituant insaturé (le Vollprädikat-Glied « est

${ }^{1}$ E. Husserl, $L U$, IV. Unt., § 10, Hua XIX/1, 326 (trad. fr., $R L$ II/2, p. 111-112).

${ }^{2}$ E. Husserl, Alte und neue Logik, Hua Mat. VI, 106 et 194-195.

${ }^{3}$ E. Husserl, Logik 1917/18, § 23b, Hua XXX, 106 : " La proposition antécédente [scil. dans une proposition hypothétique] a pour matière propositionnelle "A est b" ; elle dit "si l'on suppose que A est b". Là, nous avons à nouveau une césure : "A / est b”. » 
$\mathrm{p} »)^{1}$ ! Or, telle n'est pas la voie sur laquelle il s'engage; la copule est ne désigne pas la synthèse entre l'unité de sens saturée $S$ et l'unité de sens insaturée « est $p$ » (selon le modèle fonctionnel frégéen) mais, très classiquement, la synthèse entre sujet et prédicat :

Il faut cependant remarquer que le « est $p$ » ne peut valoir pour nous comme une représentation [scil. un syntagme, un matériau syntaxique], comme si deux représentations « $S$ » et « est $p$ » parvenaient à l'unité ; la synthèse n'a pas ici lieu entre le niveau inférieur [Unterstufe, i. e. S] et le niveau supérieur [Oberstufe, i. e. das Ausgesagte « est $p$ »], mais entre $S$ et $p^{2}$.

5/ Ce modèle prédicatif traditionnel trouve une justification dans la structure du jugement négatif.

Il existe en effet trois manières de présenter un jugement négatif : « $S$ n'est pas $p$ », « $S$ est un non- $p$ », « il est faux que $S$ soit $p$ ». Dans la première tournure, on refuse un prédicat à un sujet (ein Prädikat absprechen); dans la seconde, on attribue au sujet le prédicat nié (das negierte Prädikat zusprechen), en incorporant la négation à la signification ou au concept du prédicat $^{3}$; dans la troisième, enfin, on se tourne contre le prédicat $p$ ou, plus exactement, "contre le poser-comme- $p$ de $S$, donc contre la position de prédicat ${ }^{4}$, dans une antithèse. Or, de ces trois expressions de la négation, $\mathrm{y}$ en a-t-il une qui soit plus originaire et plus adéquate ? Quelles en sont les conséquences relativement à la structure prédicative ?

L'argumentation de Husserl est double.

Tout d'abord, des deux premières formes, la première est la plus originaire, tandis que la deuxième en est dérivée : «le prédicat conceptuel négatif implique la possibilité idéale du jugement négatif»; le concept négatif «non- $p$ » n'est pas une forme originaire, mais se laisse dériver de la proposition négative « $S$ n'est pas $p$ » en incorporant après coup au prédicat la négation; dans l'ordre de dérivation, la négation propositionnelle (copulative) précède la négation conceptuelle ${ }^{5}$. Quelle en est la conséquence pour la structure prédicative ? On pourrait s'attendre à ce que la deuxième

${ }^{1}$ E. Husserl, Alte und neue Logik, Hua Mat. VI, 195 : « Le “est” appartient ici au prédicat [das "ist” gehört hier zum Prädikat]».

${ }^{2}$ E. Husserl, Alte und neue Logik, Hua Mat. VI, 106.

${ }^{3}$ E. Husserl, Alte und neue Logik, Hua Mat. VI, 194.

${ }^{4}$ E. Husserl, Logik 1917/18, § 29a, Hua XXX, 134 : « Ich wende mich gegen das $p$, nämlich gegen das Als-p-Setzen des $S$, also gegen die Prädikatsetzung. »

${ }^{5}$ E. Husserl, Alte und neue Logik, 194. Dans le cas contraire, dit Husserl, on aurait un regressus in infinitum; nous ne comprenons pas pourquoi.

71 
tournure ( S $S$ est un non- $p$ ») soit plus favorable au primat de la structure prédicative traditionnelle: si la négation est incorporée au prédicat, elle laisse en effet intacte la forme prédicative " $S$ / est / (non- $p$ ) »; tandis que si elle affecte la copule, on obtient deux formes de copule, l'une affirmative, l'autre négative, qui peuvent s'exprimer sous la forme fonctionnelle «être $p$ » et « ne pas être $p$ ». Telle n'est cependant pas la conclusion de Husserl ! Dans la forme primitive, dit-il, ce n'est pas le nicht qui appartient au prédicat conceptuel, mais le ist nicht qui appartient au prédicat complet (ganzes oder volles Prädikat) ; le nicht n’y est pas dirigé contre la représentation-sujet $S$, mais contre le est $p$, à savoir l'identification prédicative de $S$ avec $p$; la structure fondamentale de la négation n'est donc pas une copule négatrice (verneinende Kopula), mais une copule niée (verneinte Kopula) ${ }^{1}$, une identification prédicative niée. Par conséquent, la structure originaire demeure la forme prédicative affirmative « $S$ / ist / $p$ », dont la déclinaison négative est la forme dérivée « $S$ / nicht / ist / $p »^{2}$.

Cet argument est renforcé par la confrontation entre la première et la troisième formule.

La première semble conforme à la conception traditionnelle : la forme fondamentale (Grundform) de la proposition serait la jugement catégorique, qui se scinderait en deux formes fondamentales et co-originaires (gleichursprünglich) de la prédication, l'affirmative « $\mathrm{S}$ est $\mathrm{p}$ » et la négative " $\mathrm{S}$ n'est pas $\mathrm{p}$ ". Partant du sujet, on aurait deux thèses distinctes : une thèse fondamentale (Grundsetzung) ou inférieure (Untersetzung), à savoir la position nominale du sujet, puis une thèse superstructurelle, à savoir la position du prédicat par la césure copulative, qui pourrait être affirmative ou négative $^{3}$. Or telle n'est pas la conception adéquate! La négation n’est pas

${ }^{1}$ E. Husserl, Alte und neue Logik, Hua Mat. VI, 196.

${ }^{2}$ L'argument de Husserl n'est ni très clair, ni probant. Dans sa Recherche logique portant sur «La négation », Frege a apporté bien davantage de clarté : en refusant l'assimilation traditionnelle de la négation à un acte noétique de "séparation » du sujet et du prédicat, pour la concevoir comme un élément du sens idéal («Die Verneinung », Beitr. zur Philos. des deutschen Idealismus, 1918/19, 147-149; trad. fr. in Écrits logiques..., p. 200-203) ; en invoquant le principe d'économie de pensée pour refuser le dédoublement de la négation en négation intra-propositionnelle (152-155 ; trad. fr., p. 207-209), appartenant au contenu de la pensée (au sens conceptuel), et en négation portant sur la proposition ; et en l'assimilant, au fil conducteur du principe duplex negatio affirmat, à un opérateur convertissant une proposition donnée en sa contradictoire (148 et 156-157 ; trad. fr., p. 202 et p. $212-$ 213).

${ }^{3}$ E. Husserl, Logik 1917/18, § 29a, Hua XXX, 134. 
une forme coordonnée à l'affirmation, mais une opération seconde et subordonnée: c'est une anti-thèse, un acte de se tourner après coup contre une thèse originairement affirmative, c'est-à-dire de se représenter la thèse « $S$ est $\mathrm{p}$ » pour lui opposer ensuite un «non! ». La pensée négative n’est donc pas d'une seule couche (einschichtig) comme l'est l'affirmative, mais s'avère stratifiée (geschichtet) et d'un degré de complexité plus grand ${ }^{1}$. On s'attendrait donc à ce que Husserl privilégie la troisième tournure, qui dirige la négation contre la pensée entière « $S$ est $p$ ». Et pourtant non :

Il faut prendre garde au fait que le «ne... pas...» ne se tourne pas contre la proposition entière « S est $p$ », comme si cette pensée était « rejetée » dans le jugement catégorique négatif, et "reconnue » dans l'affirmatif. Le «ne... pas... » réside de manière tout à fait exclusive du côté du deuxième constituant fondamental du jugement, à savoir le constituant prédicatif [Prädikatglied $]^{2}$.

La première forme ( $S$ n'est pas $p$ ») se révèle donc plus originaire que la troisième ( «il est faux que $S$ soit $p$ ») : la négation affecte avant tout la forme fondamentale de la prédication « est », dont elle consigne le primat.

Ajoutons à cela une remarque décisive : si les deux formes sont équivalentes du point de vue de la vérité, elles n’ont pas la même signification. «Platon n'était pas le précepteur d'Alexandre » n’a pas la même signification que "Ce n'est pas Platon qui était le précepteur d'Alexandre », la fonction discursive de cette dernière formule étant de rectifier la supposition initiale selon laquelle Platon aurait eu ce rôle ${ }^{3}$. Ainsi se clarifie le sens du « principe de tous les principes » qui prescrit le recours phénoménologique à l'évidence donatrice, censée fonder ici le primat traditionnel de la structure prédicative : l'évidence en question, c'est ici la donation des significations ; or, ces dernières se donnent dans une attitude attentive aux intentions discursives, au contexte d'énonciation d'une proposition. Si la structure prédicative traditionnelle jouit d'un primat dans le domaine de la signification, c'est donc à la condition de ne pas se limiter à la considération noématique des sens idéaux et à la valeur de vérité des propositions, mais de replacer le discours dans le cadre de ses intentions vives, dans l'élément du vouloir dire attentif aux inflexions du discours. Frege voulait affranchir la logique de la grammaire, débarrasser le langage de son écorce grammaticale pour en atteindre le noyau logique, c'est-à-dire les structures nécessaires à

${ }^{1}$ E. Husserl, Logik 1917/18, § 29a, Hua XXX, 134-135.

2 E. Husserl, Logik 1917/18, § 29a, Hua XXX, 135.

${ }^{3}$ E. Husserl, Logik 1917/18, § 29a, Hua XXX, 135. 
l'énonciation de la vérité et de la fausseté ${ }^{\text {; }}$ à l'inverse, Husserl ne se limite pas au noyau logique, mais centre l'analyse dans le domaine de l'écorce linguistique et de la parole vive, de l'écorce grammaticale en tant qu'elle est le miroir des intentions vives du vouloir dire.

6/ De là résulte une critique frontale de la décomposition frégéenne de la proposition en éléments insaturé et saturé, fonction conceptuelle et argument objectal, témoignant d'une position conservatrice en logique, qui s'oppose à tout traitement en termes de calcul fonctionnel :

[...] il n'est pas vrai que tout jugement ait des arguments, qu'il implique en soi une fonction qui, pour ainsi dire, serait dotée de valeur selon sa validité universelle ou singulière. Il existe des jugements sans argument, nous les appelons jugements fixes [feste Urteile], p. ex. «Bismarck fut le plus grand homme d'État du XIX ${ }^{\mathrm{e}}$ siècle ». Les jugements qui contiennent une fonction, nous les appelons jugements fonctionnels [Funktionsurteile]. Les premiers, par référence à la logique de l'extension, peuvent être appelés quantifiants [quantifizierende], les derniers en revanche non-quantifiants ${ }^{2}$.

Cet argument prend place dans une considération sur la conception traditionnelle de la quantification, avec sa division en trois types co-originaires de jugements : universels, particuliers, singuliers. Cette conception implique deux présupposés : d'une part l'interprétation extensionnelle de la généralité universelle, qui la reconduit à la généralité sur un ensemble d'individus conçu comme ensemble clos («tous les A»); d'autre part, le fait que le jugement singulier ( " cet A... ») soit mis sur le même plan que les autres. Or, cette division ternaire masque pour Husserl une partition binaire plus fondamentale, qui oppose les jugements fixes aux fonctionnels. Les jugements fixes impliquent une position infrastructurelle (Untersetzung) de sujet puis, édifiée sur elle, une position superstructurelle (Daraufsetzung) de prédicat grâce à la copule prédicative : dans «l'Empereur a rendu visite au prince Henri » et « Berlin est une grande ville », les sujets « l'Empereur » et «Berlin» sont posés avant la prédication, au sens où la signification nominale ou le Subjektbegriff renvoie à un Subjektgegenstand dont on

1 G. Frege, «Gedankengefüge », Beitr. zur Philos. des deutschen Idealismus 3, 1923/26, 46 : "Ma tâche est ici de dégager comme noyau logique, en écartant l'accessoire, l'emboîtement de deux pensées » (trad. fr. in Écrits logiques..., p. 227) ; "Über Sinn und Bedeutung », Ztschr. f. Philos. u. philos. Kritik, NF 100, 1892, 36 (trad. fr., p. 112); "Logik» et "Logische Allgemeinheit», in Nachgelassene Schriften, 155 et 279 (trad. fr., p. 168 et 306).

${ }^{2}$ E. Husserl, Logik 1917/18, § 32e, Hua XXX, 149. 
affirme quelque chose ${ }^{1}$. Prenons en revanche la proposition « un triangle équilatéral est équiangle », que la logique traditionnelle analyse comme réductible à la proposition universelle «tout triangle équilatéral est équiangle ", valide sur l'ensemble total des triangles équilatéraux : on n'a alors aucune position préalable de triangles équilatéraux, mais une simple fonction non thétique ou dépourvue de position (setzungslose Funktion), qui est affectée par le caractère du " en général »; l' «un » de " un triangle » fonctionne ici comme une place vide (Leerstelle) désignant un argument quelconque (quidam-Glied) qui est porteur de la quantification universelle du en général ${ }^{2}$ — "tout x qui est un triangle équilatéral est équiangle », ou « quel que soit x, s'il est un triangle équilatéral, alors il est équiangle ».

La distinction entre ces deux types de jugement est manifeste dans les jugements universels, qui doivent être divisés en deux classes : pensées de la totalité (Allheitsgedanken) et pensées de la généralité, de l'universel en général (Gedanken der Allgemeinheit, des Universellen überhaupt) ${ }^{3}$. Les jugements de totalité sont ceux qui, se rapportant à un ensemble clos d'individus, affirment que tous les individus de l'ensemble possèdent une propriété : dans les propositions «tous les arbres de cette forêt sont des sapins » et « toutes les fleurs de mon jardin sont des roses », la prédication se réfère à une totalité fermée et dénombrable de singularités, de sorte que la proposition générale est ici équivalente à une conjonction de propositions singulières ; c'est un jugement fixe, qui implique la position préalable de l'ensemble d'individus considéré. En revanche, dans une proposition mathématique comme « tout [jedes] triangle a la somme de ses angles égale à deux droits ", le jedes exprime le caractère de loi générale (Gesetzcharakter), qui vaut pour tout triangle quelconque ; mais ce quelconque ne se réfère alors à aucune totalité close de triangles qui pourrait être donnée dans une représentation unitaire, et qu'il faudrait préalablement poser dans une Untersetzung, car

un ensemble de triangles dans lequel ne manque aucun triangle ne se laisse pas reconduire à la donation. C'est un non-sens. [...] une totalité de triangles,

${ }^{1}$ E. Husserl, Logik 1917/18, § 32d, Hua XXX, 148. Même dans " un homme a sonné », on a la position d'une dénotation-sujet, bien que celle-ci demeure indéterminée.

${ }^{2}$ Ibid.

${ }^{3}$ E. Husserl, Logik 1917/18, § 39, Hua XXX, 166. 
une totalité de nombres, cela — si nous maintenons le sens de totalité — n'est pas à donner intuitivement, et n'est pas donnable dans l'intuition ${ }^{1}$.

La proposition universelle est ici fonctionnelle, et non fixe : elle n'implique aucune position du sujet collectif qu'est l'ensemble, dans la mesure où l'infinité non dénombrable excède toute capacité de représentation unitaire d'une totalité ; la place vide, ne supportant pas ici de position, est l'argument d'une proposition fonctionnelle porteuse d'une quantification universelle. $L a$ pensée de l'universalité nomologique est donc irréductible à une généralité extensionnelle sur un ensemble donné d'objets. Cette dernière possède uniquement la fonction d'auxiliaire second et commode, dont on peut retracer l'ordre de dérivation : de l'évidence universelle «tout A est B », on passe à "des A quelconques en général sont $\mathrm{B}$ ", puis à " dans la totalité des A, il n’en est aucun qui ne soit $\mathrm{B}$ », enfin à « dans une généralité universelle, une pluralité de $\mathrm{A}$ est une totalité de $\mathrm{A}$ qui ont la propriété $\mathrm{B} »^{2}$; la loi générale est alors exprimée de façon purement extensionnelle, comme simple inclusion d'un sous-ensemble dans un ensemble donné, mais ce n'est là qu'un schéma ensembliste commode, bien que dépourvu de validité intuitive.

Quelle conclusion en tirer?

Que, contrairement à la thèse frégéenne, tout jugement ne se réduit pas à l'articulation entre une fonction conceptuelle et un argument; cela ne vaut que pour les jugements de subordination d'un concept à un autre, qui ne sont pas simples, mais composés (puisque comportant une implication), mais non pour les feste Urteile, qui peuvent être des jugements d'identification (« ceci est un corbeau ») ou de subsomption d'un objet sous un concept (« Berlin est une grande ville »).

7/ En réalité, l'argument de Husserl est faible: que le domaine de définition d'une fonction soit fini ou infini (dénombrable ou non), que la totalité des objets considérés soit une totalité susceptible d'être donnée à la perception ou une totalité idéale non représentable, cela ne change rien à la nature fonctionnelle du concept. La distinction décisive n’oppose pas ici les

${ }^{1}$ E. Husserl, Logik 1917/18, § 39, Hua XXX, 168-169. Frege avait déjà développé ce point sur l'affranchissement de la généralité des propositions vis-à-vis de toute proposition singulière : cf. G. Frege, "Die Logik in der Mathematik », in Nachgelassene Schriften, 230 : «On ne doit pas penser que je cherche à asserter quelque chose sur le chef d'une tribu située au fin fond de l'Afrique et qui m’est totalement inconnu, quand je dis "tous les hommes sont mortels". Je ne suis pas en train de dire quelque chose de cet homme-ci ni de cet homme-là ; je suis en train de subordonner le concept homme au concept de ce qui est mortel » (trad. fr., p. 253).

${ }^{2}$ E. Husserl, Logik 1917/18, § 39, Hua XXX, 170. 
totalités effectives et les fictives, mais les variables liées - considérées sur un domaine de définition - aux variables libres - dépourvues d'adhérence à un domaine. Aussi l'essentiel ne réside-t-il pas ici dans la preuve ou dans le recours à une évidence phénoménologique irréfutable, mais dans l'intention de Husserl : pourquoi est-il donc important de conserver la structure prédicative traditionnelle?

En vertu de la corrélation noético-noématique, aux types idéaux de Verbundenheit syntaxique entre sujet et prédicat (exprimés par la copule « est») doivent correspondre des types idéaux d'actes ou de formes de pensée :

Dans les formes des significations de pensée [Denkbedeutungen] se réfléchissent [spiegeln sich... wieder] les formes de l'intentionnalité du penser ${ }^{1}$.

À la pluralité noématique de significations formelles (Formbedeutungen) correspond la pluralité noétique des fonctions de pensée (Denkfunktionen), des formes d'intentionnalité qui se réfléchissent dans la grammaire: identification de deux individus, attribution d'une propriété à un individu, expression d'une loi universelle sur un ensemble infini, etc. S'il est essentiel de conserver le est comme articulation fondamentale de la proposition, c'est pour désigner l'invariance noétique de la synthèse comme forme originaire de la conscience (Urform des Bewußtseins), tout en spécifiant les différents types noématiques d'articulation syntaxique en lesquels se réfléchissent les différents modes du vouloir dire - de l'acte de vouloir énoncer quelque chose d'un ou plusieurs objets. La structure prédicative exprime l'orientation de la conscience sur un ou plusieurs objets pris pour thème d'intérêt et d'énonciation; et ses déclinaisons syntaxiques sont les fils conducteurs transcendantaux qui permettent de ressaisir, dans les articulations de la parole vive, la morphologie des actes synthétiques du Denken.

La grammaire du « est » est la voie d'accès à la morphologie noétique des actes de pensée.

\section{Passage à la phénoménologie génétique : vers une genèse des formes syntaxiques?}

Quel changement le point de vue de la phénoménologie génétique apporte-til aux précédentes analyses ? La généalogie de la logique conduit-elle à

${ }^{1}$ E. Husserl, Logik 1917/18, § 19d, Hua XXX, 88. 
renverser la hiérarchie entre formes noématiques et noétiques, et à accorder aux fonctions noétiques de synthèse une efficacité productrice vis-à-vis des catégories et formes syntaxiques ? Le concept d'origine doit-il désormais s'entendre non plus comme reconduction à l'évidence, mais comme une véritable production des catégories par les actes de la conscience ?

La perspective génétique conduit à une triple relativisation de la question de l'origine du jugement prédicatif, qui la réfère à un contexte plus large.

1/ Les considérations précédentes étaient ordonnées par le concept de fondation (Fundierung) ou de stratification (Schichtung) de la logique. La logique formelle comprend les lois formelles de la syntaxe pure - qui garantissent contre le non-sens formel, c'est-à-dire contre l'enchaînement anarchique d'éléments de sens, non producteur de signification unitaire - et celles de la validité (Geltung) ou non-contradiction - qui garantissent contre le contresens formel, c'est-à-dire contre la contradiction analytique de formes propositionnelles - ; et au-delà de ces strates formelles se trouve la noétique, qui thématise les différences entre évidence et visée vide ${ }^{1}$. Ces strates sont ordonnées par le rapport de fondation : d'une part, il est possible de dégager les lois purement syntaxiques d'enchaînement sensé en faisant abstraction du niveau de la validité ; inversement, le dégagement des lois de la non-contradiction et de l'implication analytiques présupposent le respect des lois de la syntaxe ; de même, l'intuition de l'objet catégorial suppose le respect des lois de la syntaxe et de la non-contradiction, qui peuvent être dégagées en faisant abstraction d'elle.

Or, l'analyse cesse désormais de se centrer sur la seule conscience de signification (sémantique ou syntaxique), c'est-à-dire sur les deux couches formelles de la logique : il y a, d'abord, subordination téléologique du problème des structures syntaxiques à celui de la possibilité de la connaissance des objets. En effet, les formes propositionnelles de la pensée judicative et les lois de la non-contradiction formelle ne sont que des conditions formelles de possibilité de la vérité : elles n’ont de fonction qu'en vue de la connaissance d'objets et sont subordonnées à son atteinte ${ }^{2}$; à ces conditions

${ }^{1}$ E. Husserl, Logik 1917/18, § 18, Hua XXX, 77-80 : « La logique formelle dans sa stratification binaire [Zweischichtung] et comme pièce fondamentale d'une doctrine de la science. » De même Form. u. transz. Logik, §§ 13-15, Hua XVII, 54-61 (trad. fr., p. 71-79).

${ }^{2}$ E. Husserl, Erf. u. Urt., § 3, p. 8 : « [...] son intérêt [scil. celui du logicien] est dirigé sur les lois de formation des jugements - les principes et règles de la logique formelle —, non en tant que simples règles du jeu, mais en tant que règles auxquelles 
formelles doit s'ajouter, pour que la connaissance atteigne son but, l'évidence (Evidenz, Einsichtigkeit) de l'objet ${ }^{1}$. Du point de vue de la connaissance, la signification propositionnelle " $S$ est $p$ » se réduit à une nécessaire médiation conduisant à l'évidence de l'état de choses "que $S$ est bien $p$ » comme objet intentionnel de degré supérieur, ainsi qu'à l'explicitation des prédicats inhérents à l’objet primitif $S$.

Et ce primat téléologique de la connaissance de l'objet est redoublé par un primat archéo-logique relativement aux formes de jugements, lequel tend à inverser l'ordre de fondation entre sens et vérité, compréhension et évidence - renversement qui possède une portée tout à fait décisive. En effet, loin que la pure et simple compréhension du sens propositionnel constitue un niveau fondateur et autonome sur lequel s'édifierait par surcroît la possibilité de vérité ou d'intuition, c'est à l'inverse le pur et simple juger (bloßes Urteilen) qui se réduit à une modification intentionnelle dérivée du juger connaissant (erkennendes Urteilen) ${ }^{2}$. Le jugement évident, donateur de son objet, jouit d'un caractère originaire vis-à-vis du jugement non-évident, c'est-à-dire de la simple compréhension du sens propositionnel sans intuition de l'objet. C'est là la traduction, en termes génétiques, de la thèse plus ancienne selon laquelle le dégagement de la pensée (en termes techniques, de la matière ou du contenu) propositionnelle (Satzgedanke, -materie, -inhalt) s'effectue en neutralisant la position judicative ou le caractère thétique, c'està-dire en se transposant dans un acte de quasi-jugement, ou encore en substituant, à l'acte de juger, celui de la simple compréhension ; le contenu propositionnel dépourvu de caractère thétique est donc une "Idée dépendante $»^{3}$. Le premier sens de l'Ursprungsklärung, c'est par conséquent de rapporter les formes de signification aux types de jugements évidents sur les

doit satisfaire la constitution des formes à travers lesquelles peut s'instituer une connaissance en général. » (trad. fr., p. 17-18).

${ }^{1}$ E. Husserl, Erf. u. Urt., § 3, p. 8 : «Cet aperçu nous contraint à poser la question suivante : qu'est-ce qui doit s'ajouter aux conditions formelles de possibilité de la vérité pour qu'une activité de connaissance atteigne son but ? Ces conditions supplémentaires se trouvent du côté subjectif, et concernent les caractères subjectifs de l'intuition intellectuelle, de l'évidence [die subjektiven Charaktere der Einsichtigkeit, der Evidenz], ainsi que les conditions subjectives de son atteinte. » (trad. fr., p. 18).

${ }^{2}$ E. Husserl, Erf. u. Urt., § 5a, p. 15-16 : « [...] le simple juger est une modification intentionnelle du jugement connaissant. Un jugement qui fut produit dans l'évidence originaire, une connaissance qui fut obtenue une première fois dans une intuition intellectuelle peuvent assurément toujours être répétés sans la clarté de cette intuition, encore que dans une certaine distinction. » (trad. fr., p. 25).

${ }^{3}$ E. Husserl, Logik 1917/18, § 11, Hua XXX, 54-56. 
objets, d'effectuer une reconduction des significations à l'évidence des états de choses.

La conséquence essentielle en est l'impossibilité d'autonomiser la logique formelle (ainsi que le domaine de la conscience de signification) pour couper ses liens téléologiques et généalogiques avec l'intention cognitive : aucun niveau de l'analyse intentionnelle ne peut être radicalement dissocié de ses soubassements archéologiques et prolongements téléologiques. Les formes de Denkbedeutungen et de Denkakte sont donc désormais rapportées aux actes de synthèse mis en jeu dans la connaissance de l'objet : ce qu'a mis en évidence l'analyse phénoménologique effectuée dans la couche spécifique du logos idéal - à savoir l'indépendance des formes catégoriales de la signification et des lois analytiques-formelles de la validité vis-à-vis de la connaissance matériale - se voit désormais mis en question par l'élargissement de l'analyse intentionnelle au logos au sens large, qui prend désormais en compte la relation entre les différentes couches d'actes.

Est-ce à dire pour autant que les formes de la conscience connaissante soient à l'origine des formes syntaxiques ? Qu'il y a un primat des fonctions noétiques, et une production (Erzeugen) des structures syntaxiques par ces dernières ?

2/ L'Ursprungsklärung possède un second sens : tout acte de juger porte sur un objet, un thème ou substrat, et tout acte de pensée judicative véritable sur un objet présuppose la donation préalable de cet objet; l'évidence prédicative de l'état de choses "que $S$ est $p$ » implique et présuppose l'évidence antéprédicative qui est donatrice de l'objet-substrat thématique $S^{1}$. Par conséquent, les formes de jugements du logicien ne sont pas seulement des Wahrheiten an sich purement analytiques, mais doivent pouvoir se remplir par des matières syntaxiques, donc avoir un champ d'application effectif, c'est-à-dire valoir pour la connaissance d'un monde de substrats précédant la mise en forme prédicative ${ }^{2}$. Il y a donc un double rapport de la connaissance logique (prédicative) à la connaissance antéprédicative: d'une part, un rapport de fondation (Fundierungsverhältnis), au sens où la formation des catégories logiques s'édifie sur le

${ }^{1}$ E. Husserl, Erf. u. Urt., § 4, p. 11 : « [...] si l'effort de connaissance se dirige sur l'étant, si c'est l'effort pour formuler dans un jugement ce qu'il est et comment il est, il faut bien que l'étant soit déjà pré-donné [schon vorgegeben sein]. Et vu que l'acte de juger requiert quelque chose de “sous-jacent” à propos duquel il juge, un objet-ausujet-duquel, l'étant doit donc être pré-donné de telle sorte qu'il puisse devenir objet de jugement. » (trad. fr., p. 20-21).

2 E. Husserl, Form. u. transz. Logik, § 92a, Hua XVII, 230-232 : « Référence de la logique traditionnelle à un monde réal » (trad. fr., p. 300). 
soubassement du monde pré-donné à la perception ${ }^{1}$; d'autre part, un rapport téléologique d'application (Anwendung), au sens où les formes analytiques doivent servir d'instrument à la connaissance du monde des archi-objets perceptifs $^{2}$. Il est ainsi nécessaire d'étudier le mode de construction ou d'édification (das Wie des Sich-aufbauens) de l'évidence judicative (Urteilsevidenz) sur le soubassement structurel de l'évidence donatrice des objets (gegenständliche Evidenz) : comment les synthèses de degré supérieur (en particulier, celle de la prédication) s'édifient-elles sur les synthèses propres à l'évidence immédiate des objets perceptifs ${ }^{3}$ ? Leur structure dépend-elle de celle qui caractérise ces dernières ? Ou bien est-elle déterminée par les structures ontologiques des objets donnés dans la perception ? Telle est la question fondamentale de la logique, comprise en dernière instance comme étant une logique mondaine (Weltlogik) ${ }^{4}$.

3/ Ce deuxième sens du concept d'origine implique une méthode d'élucidation régressive : il s'avère nécessaire de régresser en deçà de l'acte de formalisation propre à la logique formelle.

Si en effet les évidences logiques, dans la mesure où elles sont purement analytiques, laissent les matériaux syntaxiques indéterminés en les remplaçant par des variables littérales (formes de substrat $S$, $S$ ', formes de prédicat $p, q$, formes de relation $R, R^{\prime}$, etc.), il ne faut pas prendre les formes analytiques comme possédant une validité intemporelle, mais les replacer dans l'histoire du sens (Sinngeschichte), c'est-à-dire dans l'ordre génétique

${ }^{1}$ E. Husserl, Erf. u. Urt., § 4, p. $13:$ : [...] ce rapport de fondation [Fundierungsverhältnis] concerne [...] tout jugement prédicatif évident possible en général, donc également les jugements du logicien lui-même » (trad. fr., p. 22).

${ }^{2}$ E. Husserl, Erf. u. Urt., § 4, p. 13 : « [...] ces jugements non plus n’ont pas pour contenu des "vérités en soi" flottant dans l'air mais, par leur domaine d'application [Anwendungsbereich], se réfèrent à un "monde" de substrats " (trad. fr., p. 20) ; Form. u. transz. Logik, § 83, Hua XVII, 212-213 : « [...] tout jugement concevable a en dernière instance une relation à l'objet (relation réale en un sens très large) qui est une relation à un individu [...]. Mais le sens de cette possibilité de détermination implique aussi l'applicabilité [Anwendbarkeit] à des objets arbitrairement choisis, doués de contenu matérial » (trad. fr., p. 276-277).

${ }^{3}$ E. Husserl, Erf. u. Urt., § 4, p. 14 : «L'évidence qui donne l'objet [Gegenständliche Evidenz] est la plus originaire, parce que c'est elle qui rend possible l'évidence judicative, et que l'élucidation de l'origine du jugement prédicatif doit rechercher la façon dont l'acte de juger prédicatif évident s'édifie sur l'évidence d'objet - et ce, au premier chef, pour les opérations les plus primitives de l'acte de juger prédicatif. » (trad. fr., p. 24).

${ }^{4}$ E. Husserl, Erf. u. Urt., § 9, p. 36 (trad. fr., p. 44) ; Form. u. transz. Logik, § 92b, Hua XVII, 232-234 (trad. fr., p. 303).

81 
des transformations opératoires des formes du sens. Ainsi, parmi les substrats logiques, on doit distinguer entre ceux qui portent en eux le résultat sédimenté d'une mise en forme syntaxique antérieure dont ils sont dérivés par nominalisation, et les substrats ultimes ou originaires (letzte, ursprüngliche Substrate), objets individuels qui ne portent pas en eux la trace de jugements antérieurs ni d'actes de nominalisation de formes syntaxiques. Par exemple, la généralité sensible « le rouge » est dérivée, par nominalisation, du jugement "ceci est rouge" porté sur un objet sensible perçu et de l'adjectif "rouge » qui y figure ; de même, la généralité relationnelle "la ressemblance » est dérivée du jugement "ceci ressemble à cela » et de la relation à deux termes « semblable à... » qui y figure. Et tous les jugements perceptifs de cette sorte, ainsi que les formes adjectives et relationnelles de la généralité, renvoient en dernière instance à cette maison, cette rose, cette bouteille..., en tant qu'objets d'expérience sensible immédiate donnant lieu à des jugements de perception ${ }^{1}$.

Quelle en est la conséquence pour l'analyse des formes logiques ?

C'est que la théorie de l'expérience des substrats individuels ultimes et de la théorie des jugements de perception possède un caractère paradigmatique pour la théorie du jugement catégorique et le concept de logos en général : paradoxalement, c’est la théorie de l'expérience antéprédicative donc infra-judicative, infra-linguistique - qui constitue le niveau premier et fondateur de la théorie du jugement ${ }^{2}$ !

S'il en est ainsi, c'est en vertu d'une présupposition essentielle : à savoir que l'acte de jugement présente la même structure fondamentale (Grundstruktur) à tous les niveaux de la connaissance et de l'activité logique, qu'il s'agisse de jugements scientifiques ou pré-scientifiques ${ }^{3}$. S'ensuit un précepte cartésien de passage du simple au complexe : la théorie

${ }^{1}$ E. Husserl, Erf. u. Urt., § 5c, p. 20 : « [...] nous devons donc encore distinguer, parmi les possibles objets ou substrats de jugement, ceux qui portent déjà en eux les dépôts d'un acte de jugement antérieur [Niederschläge früheren Urteilens] sédimentés dans leurs formes catégoriales, et les substrats effectivement originaires, objets intervenant pour la première fois dans le jugement comme substrats — les substrats ultimes [letzte Substrate]. » (trad. fr., p. 29) ; Form. u. transz. Logik, § 82, Hua XVII, 209-211 (trad. fr., p. 273-276).

${ }^{2}$ E. Husserl, Erf. u. Urt., § 6, p. 21 (trad. fr., p. 30-31). Form. u. transz. Logik, § 86, Hua XVII, 216-220 : "L'évidence de l'expérience antéprédicative comme thème premier en soi » (trad. fr., p. 282).

${ }^{3}$ E. Husserl, Erf. u. Urt., § 13, p. 59 : « [...] en disant "jugement”, on désigne une essence générale qui, en sa structure fondamentale, est la même à tous les degrés d'opérativité où elle intervient. » (trad. fr., p. 68). Cf. § 49, 241 (trad. fr., p. 246).

82 
du jugement doit commencer par les jugements les plus simples, ceux de la perception, parce qu'ils sont fondateurs pour toutes les formes complexes et qu'ils portent en eux les structures paradigmatiques que l'on retrouve à tous les niveaux de l'activité logicienne ${ }^{1}$. En outre, loin de considérer les jugements d'expérience en général, Husserl se limite au paradigme qu'est le jugement d'expérience externe, qui a pour fondement la perception des corps extérieurs $^{2}$ : même si l'attitude la plus courante n'est pas purement perceptive, mais pratique ${ }^{3}$, on forme la fiction méthodologique d'un sujet purement perceptif, non engagé dans l'évaluation pratique et l'action ${ }^{4}$, et l'on se restreint même au cas particulier de la perception statique d'un objet extérieur au repos. Pourquoi le choix d'un tel paradigme? En vertu de l'avantage méthodologique qu'il offre, celui de sa simplicité supérieure ${ }^{5}$ : deviendront en principe lisibles sur cet exemple simple les structures les plus élémentaires du jugement, parce que l'objet externe est doué de permanence, qu'au repos cette constance est saisie par une synthèse simple, et que nulle évaluation changeante ne vient la perturber.

D'où, enfin, l'ultime niveau de l'élucidation, qui reconduit le jugement de perception externe à la perception externe elle-même. Il s'agit de procéder à l'élargissement du concept de jugement à tous les actes objectivants de la sphère antéprédicative de la perception, en montrant que celle-ci implique déjà une forme d'opérativité logique et, surtout, que cette Leistung objecti-

${ }^{1}$ E. Husserl, Erf. u. Urt., § 49, p. 241-242 (trad. fr., p. 247) ; Form. u. transz. Logik, $\S 86$, Hua XVII, 216 et ici 219 : « [...] considéré en soi, le jugement évident (et, plus profondément, le jugement d'expérience) est le jugement originaire.» (trad. fr., p. 282 et ici 285).

${ }^{2}$ E. Husserl, Erf. u. Urt., § 14, p. 66 (trad. fr., p. 75).

${ }^{3}$ E. Husserl, Erf. u. Urt., § 14, p. 67 (trad. fr., p. 75).

${ }^{4}$ E. Husserl, Erf. u. Urt., § 14, p. 68-69: « [...] nous formons la fiction d'un sujet qui se comporte en sujet purement adonné à la considération [das sich bloß betrachtend verhält], et n'est pas incité à une activité pratique par l'étant dont il est affecté dans son environnement. » (trad. fr., p. 77).

${ }^{5}$ E. Husserl, Erf. u. Urt., § 14, p. 69 : «C’est assurément un précepte de méthode que de commencer en de telles analyses par le plus simple, et de s'élever seulement ensuite au complexe.» (trad. fr., p. 78). Cette référence à la troisième règle de Descartes (Discours de la méthode, II, AT, VI, 18) souligne le cartésianisme de Husserl sur le plan méthodique, que Heidegger avait dégagé dès son cours de 1923 Einführung in die phänomenologische Forschung, §§ 46 et 47, GA 17, p. 254 et 266 (trad. fr. A. Boutot, Introduction à la recherche phénoménologique, Paris, Gallimard, 2013, p. 276 et 288). 
vante constitue le fondement de l'intelligibilité de tous les actes logiques de niveau supérieur :

L'élucidation phénoménologique de l'origine du logique [...] trouve non seulement qu'une opérativité [Leistung] logique se trouve déjà dans des strates où la tradition ne l'a pas vue [...] mais surtout, que c'est précisément dans ces strates inférieures que sont à trouver les présuppositions celées sur les fondements desquelles seules deviennent intelligibles le sens et la légitimité supérieures du logicien ${ }^{1}$.

Cependant, derechef, quel est le niveau prédominant dans la genèse des formes syntaxiques ? Est-ce l'activité noétique déjà présente dans l'objectivation perceptive ? Ou sont-ce les structures ontologiques élémentaires de l'objet perçu, où l'on devrait trouver la matrice des catégories de l'ontologie formelle?

\section{Origines et légitimation de la structure prédicative}

Rappelons quelle est, pour Husserl, la structure fondamentale du jugement catégorique. Loin de voir dans la synthesis et la diairesis deux types cooriginaires et coordonnés, il souligne l'essentialité de la relation à l'objet (gegenständliche Beziehung) en décomposant le jugement catégorique en deux thèses : tout d'abord, une position fondamentale de type nominal, qui pose le Subjektgegenstand, la dénotation objectale ; puis, édifiée sur cette première thèse, une position de prédicat qui énonce quelque chose comme valant pour l'objet-substrat. L'énoncé est un jugement véritable s’il porte sur un objet posé comme sujet (Subjektgegenstand), et non seulement sur une signification figurant syntaxiquement en place de sujet (Subjektbedeutung) ${ }^{2}$. Telle est la structure stratifiée dont il s'agit à présent de rendre compte.

1/ Le niveau le plus patent de l'analyse est aussi le plus élevé : c'est l'analyse de la liaison prédicative proprement dite, telle qu'elle intervient dans la connaissance scientifique de l'objet, c'est-à-dire à un degré de

${ }^{1}$ E. Husserl, Erf. u. Urt., § 1, p. 3 (trad. fr., p. 13) ; cf. § 13, p. 62-63 : « [...] déjà dans l'orientation antéprédicative objectivante sur un étant, il faut parler d'un acte de juger, au sens large. » (trad. fr., p. 71).

${ }^{2}$ E. Husserl, Logik 1917/18, § 29a-b, Hua XXX, 134 et 138. Alte und neue Logik, Hua Mat. VI, 106-107. Aussi la proposition «l'actuel roi de France est chauve " n'est-elle pas un jugement proprement dit, puisque c'est un énoncé sans objet (gegenstandslos) (ibid., 195). 
discursivité élevé ; la structure prédicative du discours est rapportée à l'activité prédicative de la connaissance, et celle-ci, à son tour, ancrée dans la structure noétique fondamentale de la connaissance et dans le concept d'objet qui lui est corrélatif.

L'intérêt de la connaissance proprement dite, c'est de parvenir à une Feststellung des Seienden (une position ferme de l'étant en son mode d'être et son essence ${ }^{1}$ ), c'est-à-dire d'établir une fois pour toutes (ein für allemal) les déterminités du substrat, comme quelque chose qui demeure à jamais identique et à nouveau ressaisissable, comme un acquis durable (bleibender Besitz) qui vaut au-delà du présent de l'évidence actuelle et demeure intersubjectivement disponible (intersubjektiv verfügbar) et reste susceptible d'être réactivé en tout temps par quiconque ${ }^{2}$. Le concept d'objet de connaissance est donc une objectité d'entendement, en tant que substrat qui s'incorpore toutes les déterminités établies avec certitude par le kategorein et susceptibles de validité omnitemporelle et intersubjective ; il est fondé dans la volonté de connaissance (Wille zur Erkenntnis), entendue comme intérêt noétique qui n'est pas un invariant anthropologique ancré dans la nature humaine, mais s'identifie à la structure même de l'intentionnalité, qui tend vers le remplissement évident. Cette volonté de savoir est orientée sur l'enrichissement de sens (Sinnbereicherung) du substrat, c'est-à-dire l'élucidation progressive des déterminités qui appartiennent à son horizon interne indéfini et spécifient $S$ comme étant $p$, $q$, etc., mais aussi à sa pérennisation et à son intersubjectivation. C'est pourquoi l'activité prédicative possède une double détermination : d'une part, c'est une spontanéité productrice de sens (schöpferische, erzeugende Spontaneität), dans la mesure où seul l'intérêt cognitif produit l'objet catégorial comme étant $p, q, r$, etc., c'est-à-dire $S p q r$ (objet ayant incorporé ses attributs, donc mis en forme syntaxique) ${ }^{3}$; cependant, dans la mesure où les déterminités $p, q, r$ ne sont pas créées ou inventées, mais dévoilées dans l'évidence comme appartenant au substrat $S$, c'est aussi une spontanéité pro-ductrice, qui reconduit à l'évidence donatrice — laquelle, visant à dévoiler $S$ comme étant véritablement $p, q, r$, puise ces prédicats dans l'évidence donatrice. La prédication a donc sa source dans le $\delta \eta \lambda$ oṽv, l'acte de manifester ou désocculter l'objet comme étant ceci ou

${ }^{1}$ E. Husserl, Erf. u. Urt., § 47, p. 231 (trad. fr., p. 237).

${ }^{2}$ E. Husserl, Erf. u. Urt., § 47, p. 232-233 (trad. fr., p. 238-239).

${ }^{3}$ E. Husserl, Erf. u. Urt., § 47, p. 233 (trad. fr., p. 239).

85 
cela : c'est la structure même du comportement thématisant et désoccultant qui rend compte de la prédication ${ }^{1}$.

On retrouve ainsi, dans l'activité d'objectivation scientifique, la structure duale (Zweigliedrigkeit) qui caractérise la proposition prédicative : la prestation nouvelle qu'apporte la prédication consiste à effectuer la synthèse de recouvrement entre le substrat $S$ et le prédicat $p$, et à retenir fermement (festzuhalten) le prédicat comme inhérent à la teneur de sens de $S$ - un $S$ maintenu en son identité, mais enrichi, quant à son sens, d'un nouveau prédicat qui s'y incorpore ${ }^{2}$. On a donc bien ici, à la fois, la position infrastructurelle (Untersetzung) du Subjektgegenstand S, son maintien comme foyer (Brennpunkt) de l'intérêt cognitif, et la position superstructurelle (darauf gebaute Setzung), à savoir l'incorporation pérenne des prédicats à la teneur de l'objet. Cette manière de reconduire les formes syntaxiques à leur origine dans un intérêt cognitif permet de rapporter les différentes formes aux modalités spécifiques de cet intérêt noétique : ainsi, la différence entre la prédication et l'attribution (c'est-à-dire les formes « $S$ est $p$ » et $S p$ : «le ciel est bleu» et le ciel bleu) tient à la hiérarchie des déterminations, qui fait de l'une une saisie principale et de l'autre une saisie annexe ; « $S p$ est $q$ » signifie : $S$, déjà déterminé auparavant comme étant $p$, l'est à présent comme étant en outre $q^{3}$. Les structures linguistiques se fondent sur des modalités infra-linguistiques de l'intérêt.

2/ Cette dualité synthétique (Zweigliedrigkeit) de la prédication active est cependant isomorphe à la synthèse d'explicitation (Explikation) qui appartient à l'intérêt perceptif et pré-scientifique, encore vierge de toute volonté de détermination pérenne de l'objet: la structure de l'activité noétique supérieure de prédication est reconductible à celle de l'activité noétique inférieure de la considération perceptive. En ce sens, la connexion prédicative a une origine noétique, un ancrage dans les niveaux inférieurs de

${ }^{1}$ La thèse de Husserl s'avère donc fort proche de la thèse heideggérienne selon laquelle la fonction essentielle de l'énonciation prédicative réside dans le $\delta \eta \lambda$ oṽv , acte de mise en lumière, de faire voir l'objet tel qu'il est : cf. M. Heidegger, Die Grundprobleme der Phänomenologie, §§ 16a et 18a-b, GA 24, p. 256-259 et 304310 (trad. fr. J.-F. Courtine, Les problèmes fondamentaux de la phénoménologie, Paris, Gallimard, 1985, p. 220-223 et 257-262) ; Logik. Die Frage nach der Wahrheit, § 11, GA 21, 133.

${ }^{2}$ E. Husserl, Erf. u. Urt., § 50a, p. 242-243 (trad. fr., p. 247-248).

${ }^{3}$ E. Husserl, Erf. u. Urt., § 55a, p. 270-271 (trad. fr., p. 274-275).

86 
l'activité de la conscience ; elle n'est que la reprise, à un niveau supérieur d'activité, de la structure de la synthèse d'explicitation de l'objet perceptif ${ }^{1}$.

En effet, la structure duale de la proposition prédicative a son origine dans la dualité structurelle qui caractérise l'orientation propre à l'acte de considération explicitante de l'objet perceptif: constance de l'orientation noétique du regard de la conscience sur un même objet maintenu comme thème de l'attention, et chaîne polythétique de saisies singulières de moments de l'objet ${ }^{2}$. Dans la considération continue d'un objet $S$, on trouve l'unité d'un intérêt thématique prédominant pour le substrat $S$ (herrschendes Interesse an $S$ ), lequel, en tant que visée vide de ses déterminités potentielles, tend au remplissement - à l'actualisation des potentialités perceptives de l'objet, à la variation de ses modes de donnée subjectifs qui amène à l'évidence les déterminités actuelles et les propriétés constantes de l'objet. C'est cette constance de l'intérêt noétique qui fonde l'unité polythétique du procès de détermination de l'objet : parce que le même objet demeure thème de l'attention, la suite des modalités de la perception n'est pas une pluralité éclatée d'appréhensions singulières dépourvues de rapport, mais une unique « considération qui se développe, une unité de considération articulée » qui saisit chaque propriété ou déterminité " comme étant quelque chose qui est propre à l'objet $S$, qui en provient et se trouve en lui » (etwas vom Gegenstande $S$, etwas aus und in ihm $)^{3}$; une coupe de cuivre se détermine par sa rondeur globale, puis par un défaut dans une portion de sa surface, puis par son éclat, ses bosses, etc ${ }^{4}$. La forme catégoriale de la prédication se construit ainsi sur fond de synthèse antéprédicative d'explicitation, et trouve en elle son origine : c'est la fonction intentionnelle de l'intérêt cohérent pour l'objet-thème, et son remplissement continu par des modes de donnée de propriétés diverses, qui transforment $S$ en un substrat de détermination continue et les moments $p, q, r$ en prédicats déterminant $\mathrm{S}$, et qui font du est un nom pour l' «unité de recouvrement» ou de congruence (Deckungseinheit) de la synthèse progressive d'explicitation ${ }^{5}$.

L'origine de la prédication semble donc prima facie intégralement noétique: car ce sont les structures de l'acte de synthèse qui fondent les

${ }^{1}$ E. Husserl, Erf. u. Urt., § 24a, p. 124 : «La synthèse d'explicitation [explikative Synthesis] comme lieu d'origine des catégories de "substrat" et "déterminité", avec pour tâche de l'analyser » (trad. fr., p. 131).

${ }^{2}$ Ibid.

${ }^{3}$ E. Husserl, Erf. u. Urt., § 24a, p. 126 (trad. fr., p. 133).

${ }^{4}$ E. Husserl, Erf. u. Urt., § 24c, p. 130 (trad. fr., p. 137).

${ }^{5}$ E. Husserl, Erf. u. Urt., § 24a, p. 127 (trad. fr., p. 134).

87 
formes syntaxiques auquel le discours confère une expression linguistique ; les syntaxes ont leur fondement dans les structures du sujet transcendantal. Mais il ne faut pas interpréter pour autant cette thèse en un sens radicalement subjectiviste! En effet, l'intérêt explicitant n'est pas un invariant anthropologique, une tendance appartenant à la nature humaine qui devrait être admise comme une donnée factuelle, selon un geste copernicien. Il existe, au contraire, une genèse de l'intérêt à partir de l'objet et de sa structure d'horizon: loin que l'objet surgisse de façon isolée et que ses propriétés inconnues soient totalement indéterminées, il est saisi sur fond d’un monde pré-donné et typifié, ordonné en types de réalités empiriques (plantes, arbres, buissons, animaux, mammifères, poissons, etc.) — de sorte que l'objet apparaît toujours avec le caractère de familiarité (Vertrautheit), dont les propriétés inconnues sont toujours déjà anticipées en fonction du type empirique auquel il est rattaché par association ${ }^{1}$. Ce qui pousse le sujet à déployer l'horizon interne des déterminités de l'objet selon des voies épistémiques pré-tracées, c'est donc la typification empirique de l'horizon externe.

Par là se dévoile un fondement ontologique de la prédication : car si le monde de l'expérience perceptive ne se présentait pas comme un monde d'objets semblables entre eux et tendant à s'ordonner en classes de similitude, jamais ne pourrait se constituer le caractère de familiarité typique qui suscite la recognition de l'objet comme étant ceci ou cela, et qui pousse le sujet à anticiper les propriétés de l'objet et à les vérifier. Un monde anarchique d'objets qui varieraient sans cesse et seraient dépourvus de similitude frappante ne pourrait donner lieu à aucune explicitation, donc à aucune prédication ${ }^{2}$; c'est la structure ontologique stable et typifiée du monde sensible qui assure un fondement à la possibilité de la structure prédicative du discours; les structures analytiques-formelles de la syntaxe portent la trace de leur dépendance à l'égard des structures ontologiques matériales du monde perceptif.

3/ Poursuivons la généalogie de la prédication jusqu’à son niveau ultime, celui de l'expérience perceptive de singularité. Y retrouve-t-on des structures semblables? Celles-ci sont-elles de nature noétique ou ontologique?

${ }^{1}$ E. Husserl, Erf. u. Urt., § 8, p. 31-33, et § 24a, p. 124-125 (trad. fr., p. 40-43 et 131132).

${ }^{2}$ On trouve là un écho husserlien de l'objection faite à Hume par Kant à propos de la synthèse de la reproduction dans l'imagination (cf. KrV, Transz. Deduktion, A 100/101, trad. fr. DM, p. 713-714, AR, p. 180).

88 
La synthèse d'explicitation semble avoir sa préfiguration dans les structures noétiques de la perception d'objet externe. En effet, loin de se limiter au donné immédiat et actuel, l'intention perceptive " va au-delà du donné et de son mode de donnée momentané pour tendre vers un plus ultra à prolonger » (hinaus tendiert auf ein fortgehendes plus ultra) ${ }^{1}$. Si l'intention excède le donné actuel, c'est que l'objet spatial est d'emblée perçu comme objet spatial susceptible d'être donné dans une infinité de perspectives (de près ou de loin, dans telle ou telle orientation), et comme objet appartenant à un certain type déterminé par l'horizon de similitude familière. Tout donné actuel éveille ainsi, par son insertion dans la typique du monde, un horizon de potentialités perceptives, ainsi que la tendance au remplissement des visées potentielles : la donation actuelle est débordée par la potentialité, et l'affection actuelle, par la « tendance au remplissement parfait » et l' « intérêt pour l'enrichissement du "soi" de l'objet $»^{2}$. Ainsi s'opère, en langage leibnizien, le passage de la repraesentatio à l'appetitio : la donation perspective est en même temps conscience de sa propre inadéquation, ce qui motive la tendance à multiplier les perspectives sur l'objet par les kinesthèses subjectives. C'est là une pulsion perceptive qui n'a encore rien à voir avec l'activité d'explicitation ou de thématisation, mais qui en préfigure cependant les traits synthétiques : pulsion sans volonté, où la syntaxe prédicative trouve son fondement le plus profond ${ }^{3}$.

Est-ce là cependant l'origine exclusive de la prédication? La syntaxe a-t-elle son origine unique dans les structures noético-noématiques du sujet pur?

Au premier abord, il semble en être ainsi. Ainsi, par exemple, la différence entre substrat et déterminité semble purement relative à l'orientation noétique de l'intérêt. Car loin qu'il y ait des substrats et des prédicats absolus, toute activité d'explicitation peut abandonner son substrat originaire pour prendre comme nouveau substrat l'une de ses déterminités : voyant un parterre de fleurs, on peut y prélever une fleur pour en faire le foyer de son attention, puis s'intéresser à sa forme ou à sa couleur, puis à celle de son

${ }^{1}$ E. Husserl, Erf. u. Urt., § 19, p. 87 (trad. fr., p. 96-97) ; cf. § 8, p. 31 (trad. fr., p. 40) ; Cart. Medit., § 20, Hua I, 84 (trad. fr. dir. M. de Launay, Médit. cart., Paris, Puf, 1994, p. 92).

${ }^{2}$ E. Husserl, Erf. u. Urt., § 19, p. 87 : « Avec tout ce qui est effectivement donné, des horizons sont éveillés. » (trad. fr., p. 97)

${ }^{3}$ E. Husserl, Erf. u. Urt., § 19, p. 89 : « Il s’agit là de mises en œuvre de tendances perceptives, des "activités" en un certain sens, bien que ce ne soient pas des actions volontaires [obschon nicht willkürliche Handlungen]. » (trad. fr., p. 98). 
pistil, etc. ${ }^{1}$; c'est alors le parterre qui est le premier substrat relatif, puis la fleur considérée, puis sa forme, sa couleur, son pistil, qui ne possèdent qu'une indépendance relative à l'acte de considération thématique. L'acte de thématisation relativise la distinction entre concretum et abstractum (étant indépendant et moment dépendant), pour conférer à son objet une indépendance relative en le substratifiant ${ }^{2}$; il préfigure ainsi l'acte de nominalisation ou de substantification qui, d'un jugement, extrait un objet de degré supérieur. On peut, de la sorte, retracer la généalogie de la nominalisation à travers les couches noétiques: de la nominalisation à la thématisation substratifiante, puis de celle-ci à l'acte de se tourner vers...

4/ Toutefois, cette relativisation noétique a ses limites.

Le point de vue génétique, appliqué au discours, conduisait à distinguer les substrats porteurs de syntaxe implicite et les substrats ultimes, vierges de toute mise en forme syntaxique (maison, toit, fleur, etc.) ; et, parallèlement, à dégager des adjectifs ultimes (vert, coloré, dur, plat) par opposition aux adjectifs porteurs de syntaxe, ainsi que des relations ultimes (à gauche de..., plus grand que..., plus dur que...) par opposition aux relations porteuses de syntaxe. L'histoire du sens pouvait ainsi être déconstruite de manière à reconduire aux représentants ultimes de chaque catégorie syntaxique : substrats, prédicats, relations ultimes ${ }^{3}$. Or, une même opération régressive et déconstructrice peut s'effectuer dans les couches inférieures de l'explicitation et de l'expérience antéprédicatives: si tout explicatum peut être transformé par l'intérêt en substrat, il est, à l'inverse, possible de défaire la chaîne des intérêts substratifiants pour revenir « en dernière instance, et nécessairement, à des substrats qui ne sont pas issus d'une substratification ${ }^{4}$ - c'est-à-dire des substrats absolus: non plus ceux du discours (non reconductibles à une forme syntaxique antérieure et non substantive), mais ceux de l'expérience (non reconductibles à des parties, propriétés ou relations de substrats plus primitifs). Ce qui caractérise de tels substrats absolus, c'est la notion classique de substantialité, non plus entendue comme indépendance ontologique (être en soi, cause de soi) ou comme concevabilité isolée (être pensé par soi), mais traduite en termes d'expérimentabilité directe et isolée :

${ }^{1}$ E. Husserl, Erf. u. Urt., § 28, p. 147 (trad. fr., p. 153).

2 E. Husserl, Erf. u. Urt., § 29, p. 151 (trad. fr., p. 156-157).

${ }^{3}$ Husserl, Form. u. transz. Logik, § 82, Hua XVII, 210-211 (trad. fr., p. 274-275).

${ }^{4}$ Husserl, Erf. u. Urt., § 29, p. 152 (trad. fr., p. 158). 
Un substrat absolu se distingue donc par le fait que l'on peut en faire directement et simplement l'expérience [schlicht geradehin erfahrbar], le saisir immédiatement [unmittelbar erfaßbar], et qu'il est possible de procéder immédiatement à son explicitation ${ }^{1}$.

Il en est même pour les déterminités. Toute déterminité est issue d'un procès de détermination et d'explicitation d'un substrat, de sorte que lui échoit la propriété que la pensée classique attribuait aux modes, à condition de la traduire en termes d'expérience : toute déterminité est expérimentée en autre chose, à même un substrat. Cependant, il faut distinguer les déterminités dérivées et les originaires: les premières sont inhérentes à des substrats dérivés, tandis que les secondes échoient à des substrats primitifs, de sorte que la forme de déterminité leur est essentielle, en tant qu' « être-tel d'un autre être »; tel est, par exemple, le cas de la couleur ou de la forme d'un corps extérieur ${ }^{2}$. La différence entre substrat et déterminité absolus correspond donc à la distinction ontologique entre concretum et abstractum, entre étant-en-soi et étant-en-autre-chose tels qu'ils sont perçus dans l'expérience sensible :

Tout ce dont on peut faire l'expérience se caractérise ou bien comme quelque chose qui est pour soi et en soi [etwas für sich und in sich], ou bien comme quelque chose qui $n$ 'est qu'à même un autre étant, à même un étant qui est pour soi [etwas, das nur an einem anderen, an einem für sich Seienden ist [...] En ce sens, les substrats absolus sont indépendants, les déterminités absolues dépendantes ${ }^{3}$.

Quelle en est la conséquence pour la question de l'origine de la structure prédicative?

C'est que la copule est est ainsi reconduite à son ultime fondement, ontologique et non plus noétique, relatif à la structure des contenus représentés ou des objets d'expérience, et non plus aux modes de l'intérêt subjectif. Les divers modes de connexion prédicative possibles dans la proposition sont reconductibles aux diverses manières dont les contenus partiels sont inclus dans l'objet total, c'est-à-dire aux différents types de relation méréologique entre tout et partie.

${ }^{1}$ E. Husserl, Erf. u. Urt., § 29, p. 153 (trad. fr., p. 159).

2 E. Husserl, Erf. u. Urt., § 29, 156 (trad. fr., p. 162). Cf. R. Descartes, Les principes de la philosophie, I, art. 56, AT IX, II, p. 49.

${ }^{3}$ E. Husserl, Erf. u. Urt., § 29, p. 155-156 (trad. fr., p. 161).

91 
En effet, loin d'être univoque, l'identité de la copule est recouvre une pluralité de modes de connexion entre sujet et prédicat : l'inhérence (comme dans « le ciel est bleu »), la relation (comme dans « l'oiseau est à gauche de l'arbre »), l'inclusion (comme dans « la brique est une partie du toit»), etc. Toutes ces modalités renvoient à une synthèse prédicative qui possède une spécificité vis-à-vis des autres modes de synthèse : elle est toujours fondée sur le «recouvrement selon le sens de l'objet» (Deckung nach dem gegenständlichen Sinn $)^{1}$. Si, en tant que Synthesis der Überschiebung, toute synthèse doit son unité à l'activité continue d'un même ego, en revanche seule la synthèse prédicative est une synthèse d'identité (Identitätssynthesis) fondée sur l'unité noématique de l'objet: ce n'est pas une synthèse de similitude (comme l'est la ressemblance entre deux couleurs), ni une synthèse d'identité totale (comme dans le cas frégéen de l'identification de l'étoile du matin avec l'étoile du soir), mais une synthèse d'identité partielle - à savoir la congruence entre la saisie globale de $\mathrm{S}$ et les saisies partielles de $\mathrm{p}, \mathrm{q}, \mathrm{r}^{2} \ldots$

Or, qu'est-ce donc que ce recouvrement partiel (Partialdeckung) dans le cas des jugements d'expérience?

C'est la congruence selon le rapport de tout à partie, entendu au sens large. L'origine structurale de la synthèse prédicative réside alors dans la connexion entre l'objet et ses moments ou fragments constituants; et la diversité des modes de prédication provient de celle des modes de connexion entre tout et parties. Ainsi peut-on distinguer, dans un tout, entre ses parties dépendantes et indépendantes : la couleur blanche est dans le papier, au sens où elle lui est inhérente et ne peut être représentée qu'en lui, tandis que le pied d'un cendrier est dans le cendrier à titre de fragment isolable, représentable isolément; les fragments indépendants sont extérieurs les uns aux autres tout en étant en connexion entre eux, alors que les moments dépendants se compénètrent et sont donnés comme co-affectants en un même tout $^{3}$. De même, on peut distinguer entre le mode d'être dans... de la qualité et celui qui appartient à un moment dépendant immédiat : le bord ou la surface limitant une chose sont des moments inséparables de la chose, mais n'en sont pas des qualités remplissantes comme la couleur ou la rugosité ; et la forme de connexion d'une partie avec le reste de l'objet, pour être un moment dépendant, n'en constitue pas davantage une qualité ${ }^{4}$. Enfin si, en

${ }^{1}$ E. Husserl, Erf. u. Urt., § 24b, p. 128 (trad. fr., p. 135).

${ }^{2}$ E. Husserl, Erf. u. Urt., § 24b, p. 129 (trad. fr., p. 135-136).

${ }^{3}$ E. Husserl, Erf. u. Urt., §§ 30-31, p. 161-165 (trad. fr., p. 166-171).

${ }^{4}$ E. Husserl, Erf. u. Urt., § 32 a-b, p. 167-168 (trad. fr., p. 172-173). 
toute rigueur, seul le monde comme totalité absolue est un substrat originaire, en réalité tous les objets, à titre de substrats relatifs finis inclus dans le monde, sont en connexion les uns avec les autres; et tous les jugements relationnels qui explicitent leurs relations se fondent en dernière instance sur les diverses manières qu'ont les choses finies d'être dans le monde selon divers modes de connexion (temporalité, spatialité, causalité, etc.) ${ }^{1}$. Tous les modes syntaxiques de la prédication propre aux jugements d'expérience ultimes se fondent ainsi dans des modes de connexion ontologiques et antéprédicatifs, qui prescrivent par voie de corrélation des modalités d'expérience ; la grammaire pure a ses assises dans l'ontologie formelle des touts et parties, et celle-ci, à son tour, dans l'ontologie matériale des formes de connexion réales. L'ontologie des relations matériales et l'élucidation de leur mode de constitution ont une fonction déterminante pour la grammaire implicite du est.

\section{Portée et limites de la généalogie de la prédication}

Les analyses husserliennes se déploient dans une double perspective.

La première est l'élucidation des formes de signification propositionnelle, qui doit légitimer le primat traditionnel de la structure prédicative ; en définitive, c'est la réflexion noétique sur les intentions du vouloir dire, située au plus près des inflexions de la parole vive, qui permet de la défendre contre les attaques brentaniennes et frégéennes; contre la volonté frégéenne de réduire l'écorce linguistique qui masque le noyau logique, Husserl pose l'essentialité de vêtement linguistique, fil conducteur donnant accès aux modes intentionnels du vouloir dire qui s'y réfléchissent ou s'y consignent.

La seconde, plus décisive, est la généalogie de la structure prédicative : procédant à une réduction cartésienne du complexe au simple, on y régresse des jugements complexes aux jugements élémentaires de l'expérience externe, censés dévoiler les structures essentielles de tout jugement en général ; puis, régressant des jugements d'expérience à l'expérience antéprédicative, on s'efforce de ressaisir le fondement des formes syntaxiques, à la fois dans les structures noétiques de l'intentionnalité explicitante, puis perceptive, et dans les structures noématiques des contenus perceptifs.

Le discours phénoménologique est ainsi porteur d'une quadruple présupposition.

${ }^{1}$ E. Husserl, Erf. u. Urt., § 29, p. 156-158 (trad. fr., p. 162-163). 
1/ L'analyse logique ne vise pas, comme pour Frege, à purifier la structure du langage pour le subordonner aux besoins de la pensée scientifique, mais à se replacer au plus près de la parole vive et de ses intentions signifiantes en général ; en dernière instance, la logique ne se réduit pas à la seule doctrine de la science - bien qu'elle soit souvent définie en ces termes par Husserl - , mais englobe une doctrine du logos au sens large, à savoir une élucidation de la discursivité commune et de ses structures formelles.

2/ Ce sont les mêmes structures qui sont présentes dans les jugements élémentaires d'expérience et les jugements de degré élevé, notamment ceux de la pensée scientifique : l'essentiel réside dans quelques structures fondamentales (prédication, négation, modalisation), et le reste n’en est qu'une superstructure dérivable par certaines transformations opératoires ; il n’y a pas de pensée complexe, mais seulement des dérivations plus ou moins complexes de formes primitives. Par conséquent, l'élucidation de l'infrastructure que forment les structures élémentaires des jugements d'expérience perceptive doit en principe livrer l'ensemble-base de toutes les structures syntaxiques possibles, c'est-à-dire livrer l'eidos de la discursivité en général.

3/ La perception des corps en repos est prise pour paradigme de l'expérience antéprédicative où se laissent lire les structures de la syntaxe pré-linguistique qui fondent les formes de la grammaire pure : un seul terrain de l'expérience est considéré comme exemplaire pour toute expérience en général.

4/ Dans cette analyse, l'expérience antéprédicative n’est pas élucidée pour elle-même, mais en vue d'y ressaisir la préfiguration des structures logiques ${ }^{1}$ : conformément à l'orientation primitive de l'ontologie grecque, le logos demeure le fil conducteur de toute cette généalogie de la logique.

Pointons le troisième présupposé, qui est d'ordre méthodologique : estil légitime de prendre pour thème un seul terrain, et d'en faire la matrice de toute expérience ? Sans doute, si le domaine en question est premier au sein de l'expérience. Mais est-ce le cas ? Au contraire, Husserl admet avoir opéré une idéalisation et forgé une fiction méthodologique : car le plus souvent, on n'est pas installé dans la pure perception sensible de l'objet, mais engagé dans l'action et l'évaluation pratique ; " on saisit les choses comme étant

${ }^{1}$ E. Husserl, Erf. u. Urt., $\S 14$, p. 71 : « les structures du percevoir ne sont prises en considération que dans la mesure où c'est nécessaire pour comprendre comment les opérations logiques, avec les formations logiques qui en sont le résultat, s’édifient sur l'expérience perceptive sensible ; comment, sur le fondement de la perception, des objets catégoriaux [...] sont produits par la spontanéité logique. » (trad. fr., p. 7980). 
agréables, utiles, etc., avant d'avoir pu accéder, pour des raisons particulières, à un intérêt qui soit propre à la pure considération ${ }^{1}{ }^{2}$; l'horizon de familiarité du monde, qui détermine le sujet à explorer l'horizon interne des objets et à en expliciter les prédicats, n’a donc pas seulement son origine dans la praxis cognitive, mais se détermine « aussi et avant tout à partir des aspects familiers de l'étant qui dérivent de la praxis de la vie quotidienne et de l'activité manuelle ${ }^{2}$; et si le monde environnant est d'emblée un monde culturel où les étants ont des déterminités de signification culturelle, ces dernières « sont des déterminités d'une tout autre sorte que celles du corps en tant que corps ${ }^{3}$. Cela n'impose-t-il pas de se situer d'emblée dans l'attitude qui est la nôtre de prime abord et le plus souvent, et de faire de la préoccupation quotidienne le terrain d'élucidation de la généalogie de la logique?

Or, à quoi pourrait conduire une telle analyse ? À renverser le quatrième présupposé : ce n'est pas le logos qui doit être pris pour fil conducteur conduisant à l'étant de l'expérience et à ses structures ; mais inversement, loin d'être un comportement flottant en l'air, l'activité énonciative et prédicative doit être resituée au sein de l'être-au-monde et de ses modalités ${ }^{4}$. Le problème de la généalogie de la logique devient donc: comment l'énonciation prédicative dérive-t-elle du commerce quotidien avec l'étant environnant ? Et cette dérivation s'entend comme dégradation ou nivellement formalisant : car si, pour l'affairement quotidien, « le marteau est lourd », ce n'est pas au sens où le sujet-marteau a pour prédicat la lourdeur, mais au sens où il est par exemple trop lourd; le marteau s'y dévoile en tant que (als) Womit disponible, possédant une signifiance relative au commerce pratique ; c'est seulement après coup que l'énoncé arrache le marteau à ce commerce affairé pour en faire le Worüber d'un énoncé qui l'explicite et le fait voir comme lourd. Si le est de la prédication a son origine dans l'en tant que... (als), dans l'affairement quotidien, cet en tant que... demeure relatif au regard de la préoccupation pratique, qui comprend l'étant en son ustensilité significative : c'est un en tant que... herméneutique, fondé dans la structure existentiale du comprendre (Verstehen, hermeneuein) qui caractérise le

${ }^{1}$ E. Husserl, Erf. u. Urt., § 14, p. 67 (trad. fr., p. 75).

${ }^{2}$ E. Husserl, Erf. u. Urt., § 12, p. 52 (trad. fr., p. 61).

${ }^{3}$ E. Husserl, Erf. u. Urt., § 29, p. 158 (trad. fr., p. 164).

${ }^{4}$ M. Heidegger, Sein und Zeit, § 33, Halle, Niemeyer, 1927, p. 156 : «L'énoncé n'est pas un comportement flottant en l'air [Aussage ist kein freischwebendes Verhalten] [...], mais se tient toujours sur la base de l'être-au-monde [auf der Basis des In-der-Welt-seins]. » (trad. fr. E. Martineau, Etre et temps, Paris, Authentica, 1985, p. 126). 
Dasein affairé. Dans le comportement théorétique ou la visée explicitante, cet en tant que... originaire se dégrade en un en tant que... apophantique qui prend en vue l'objet comme substrat subsistant, le détermine, en explicite et en communique les prédicats ${ }^{1}$.

La prédication possède donc une triple origine. C'est, d'abord, un mode second et dérivé de l'explicitation qui convertit l'étant zuhanden dans le commerce pratique en substrat vorhanden pour le pur et simple regard : toute structure purement logique a sa provenance dans le comportement théorétique, qui opère une neutralisation de l'être-au-monde et un nivellement des pragmata en objets subsistants - fondement de l'identité apophantique. À un niveau plus profond, c'est la significativité (Bedeutsamkeit) pratique du monde environnant, où les choses se révèlent au regard de la préoccupation en tant que ceci ou cela : en tant que... herméneutique, irréductible à tout mode d'inhérence ou de connexion ontologique de parties avec un tout. C'est, enfin, le comprendre comme structure de l'exister ou de l'être-au-monde: compréhension de l'en-vue-de-quoi (Worumwillen) de l'étant environnant dévoilé au regard pratique, et de l'être de l'étant qui ne relève pas du domaine de la prédication parce que, impliqué d'emblée en toute prédication, il rend possible la prédication en général ; comprendre qui se fonde dans la compréhension, par le Dasein, de ses propres possibilités, c'est-à-dire de l'ensemble de ses manières de se rapporter à l'étant et, en définitive, de la constitution fondamentale tout entière de l'être-au-monde ${ }^{2}$. La logique trouve ainsi son fondement ultime dans l'auto-compréhension du Dasein et du comprendre ontologique : loin d'être ramenée aux structures ontologiques ultimes des objets de la perception ou des contenus de la représentation, la logique est reconduite vers les structures ontologiques de l'être-au-monde qui, pour Heidegger, constituent le fondement de l'intentionnalité.

Quel est donc le propre d'une logique phénoménologique?

C'est, d'abord, de régresser du domaine de la signification idéale vers les modes de visée, de comportement et d'évidence subjectifs propres à l'étant qui, par son discours, vise les formes syntaxiques; un premier déplacement est ainsi opéré, depuis les articulations syntaxiques de la signification vers celles de la pensée. Mais surtout, c'est d'opérer un second déplacement de l'orientation thématique: depuis le domaine de la signification idéale vers un champ infra-linguistique et pré-idéal où ce

${ }^{1}$ M. Heidegger, Sein und Zeit, § 33, p. 157-158 (trad. fr., p. 126-127) ; Logik, § 12a, GA 21, p. 143-153.

${ }^{2}$ M. Heidegger, Sein und Zeit, § 31, p. 143-145 (trad. fr., p. 118-120). 
dernier a sa source ; donc de rechercher l'origine ultime du logos dans des structures pré-logiques - formes noétiques de l'expérience perceptive, structures ontologiques de l'étant perçu, modalités de la compréhension ontologique. Ne se limitant pas à la pensée logique au sens strict, on opère un élargissement considérable de la notion de logique, pour restituer les échafaudages qui mènent à l'élaboration de la syntaxe et replacer la pensée logifiante dans le mode d'être, de comprendre et de faire-expérience du sujet ou du Dasein. Le pur logicien peut-il se satisfaire de cette reconduction de syntaxes strictement normées aux syntaxes faiblement normées de ces domaines pré-logiques? 